TRANSACTIONS OF THE

AMERICAN MATHEMATICAL SOCIETY

Volume 350, Number 4, April 1998, Pages 1619-1641

S 0002-9947(98)01912-6

\title{
$n$-UNISOLVENT SETS AND FLAT INCIDENCE STRUCTURES
}

\author{
BURKARD POLSTER
}

\begin{abstract}
For the past forty years or so topological incidence geometers and mathematicians interested in interpolation have been studying very similar objects. Nevertheless no communication between these two groups of mathematicians seems to have taken place during that time. The main goal of this paper is to draw attention to this fact and to demonstrate that by combining results from both areas it is possible to gain many new insights about the fundamentals of both areas.

In particular, we establish the existence of nested orthogonal arrays of strength $n$, for short nested $n$-OAs, that are natural generalizations of flat affine planes and flat Laguerre planes. These incidence structures have point sets that are "flat" topological spaces like the Möbius strip, the cylinder, and strips of the form $I \times \mathbb{R}$, where $I$ is an interval of $\mathbb{R}$. Their circles (or lines) are subsets of the point sets homeomorphic to the circle in the first two cases and homeomorphic to $I$ in the last case. Our orthogonal arrays of strength $n$ arise from $n$-unisolvent sets of half-periodic functions, $n$-unisolvent sets of periodic functions, and $n$-unisolvent sets of functions $I \rightarrow \mathbb{R}$, respectively.

Associated with every point $p$ of a nested $n$-OA, $n>1$, is a nested $(n-1)$ OA - the derived $(n-1)$-OA at the point $p$. We discover that, in our examples that arise from $n$-unisolvent sets of $n-1$ times differentiable functions that solve the Hermite interpolation problem, deriving in our geometrical sense coincides with deriving in the analytical sense.
\end{abstract}

\section{Flat Laguerre Planes, flat afFine Planes AND UNISOLVENT SETS OF FUNCTIONS}

Consider the incidence structure of non-vertical plane sections of the cylinder $\left\{(\cos t, \sin t, z) \in \mathbb{R}^{3} \mid t \in[-\pi, \pi], z \in \mathbb{R}\right\}$ over the unit circle $\mathbb{S}^{1}$ in the $x y$-plane (see Figure 1). The point set of this incidence structure $(P, C)$ is the cylinder $P=\mathbb{S}^{1} \times \mathbb{R}$ and its "circle set" $C$ is the set of all graphs of functions in $F$, where $F$ is the set of functions $\mathbb{S}^{1} \rightarrow \mathbb{R}: t \mapsto a+b \cos t+c \sin t, a, b, c \in \mathbb{R}$ (here we identify $\mathbb{S}^{1}$ with the interval $[-\pi, \pi)$ in the usual way). Furthermore, in this context, two points $\left(x_{1}, y_{1}\right),\left(x_{2}, y_{2}\right) \in \mathbb{S}^{1} \times \mathbb{R}$ are called parallel if and only if $x_{1}=x_{2}$. It is easy to see that $(P, C)$ satisfies the following axioms:

(L1) Three pairwise non-parallel points can be uniquely joined by a circle.

(L2) For two non-parallel points $p, q$ and a circle $c$ through $p$ there exists a uniquely determined circle through $q$ that touches $c$ at $p$, i.e., intersects $c$ only in the point $p$, or coincides with $c$.

Received by the editors October 3, 1994 and, in revised form, July 20, 1996.

1991 Mathematics Subject Classification. Primary 41A05, 51H15; Secondary 05B15, 51B15.

Key words and phrases. Non-linear interpolation, unisolvent set, Chebyshev system, Laguerre plane, Laguerre $m$-structure, orthogonal array, topological incidence geometry.

This research was supported by a Feodor Lynen fellowship. 


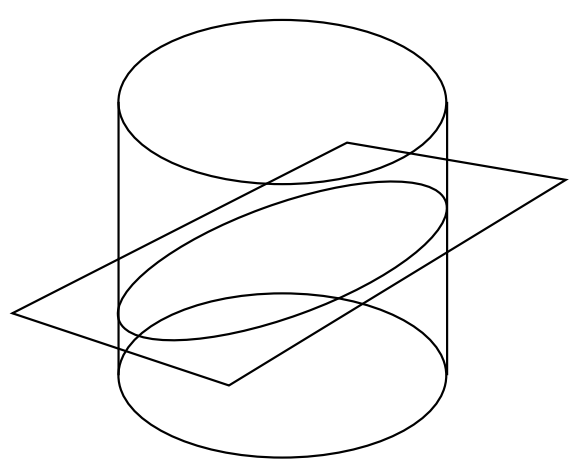

FIGURE 1

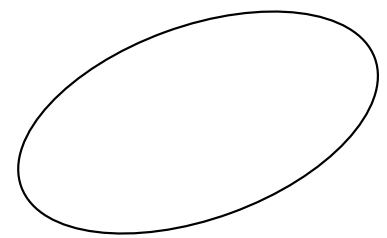

Figure 2

Incidence geometers call $(P, C)$ the classical flat Laguerre plane over the reals. People interested in interpolation theory will have noticed that axiom L1 just says that the set of continuous functions $F$ that defines the circle set is a periodic 3unisolvent set. In general, an incidence structure $\left(\mathbb{S}^{1} \times \mathbb{R}, C\right)$, where $C$ is a set of graphs of continuous functions $\mathbb{S}^{1} \rightarrow \mathbb{R}$, and which satisfies axioms L1 and L2, is called a flat (or 2-dimensional) Laguerre plane. Notice that in the above example $F=\operatorname{span}\{1, \sin t, \cos t\}$, i.e., the periodic 3 -unisolvent set $F$ is the linear span of the periodic Chebyshev system $\{1, \cos t, \sin t\}$ on $[-\pi, \pi]$. It can be shown (not hard) that a periodic Chebyshev system $\{1, u(t), v(t)\}$ on $[-\pi, \pi]$ gives rise to a flat Laguerre plane if and only if the set $\{(v(t), u(t)) \mid t \in[-\pi, \pi]\}$ is an oval, i.e., a differentiable, strictly convex, simply closed curve, in the $x y$-plane (see Figure 2 ). The associated flat Laguerre plane can then be considered as the incidence structure of non-vertical plane sections of the cylinder over this oval. This just means that in the construction described above we replace the unit circle, which is an oval, by an arbitrary oval. We note that there exist lots of flat Laguerre planes that do not arise in this manner.

Let $(P, C)$ be a flat Laguerre plane and let $p$ be a point in $P$. The derived incidence structure $\left(P_{p}, L_{p}\right)$ at the point $p$ is an incidence structure having a point set $P_{p}$ and a line set $L_{p}$. The point set consists of all points in $P$ not parallel to $p$, i.e., all points that are not contained in the same vertical on the cylinder as $p$, and the line set consists of all the circles through $p$ that have been punctured at this point. It turns out that this incidence structure is a flat dually affine plane, i.e., it satisfies the following axioms:

(DA1) Two non-parallel points can be uniquely joined by a line.

(DA2) Given any point $p$ and line $l$, there is exactly one line $l^{\prime}$ that contains $p$ and either coincides with $l$ or does not intersect $l$.

The line $l^{\prime}$ is called the parallel to $l$ through $p$. The parallelism on the line set turns out to be an equivalence relation. We identify the left and right boundaries of the "strip" $P_{p}$ with a copy of $\mathbb{R}$ such that the resulting topological space is a Möbius strip. At the same time we extend every line in $\left(P_{p}, L_{p}\right)$ by a point in this copy of $\mathbb{R}$. We can identify and extend in such a way that all lines parallel to a given line get extended by the same point and all extended lines are homeomorphic to the circle. When speaking of a Möbius strip we always have the set $[-\pi, \pi] \times \mathbb{R}$ in 
mind whose left and right boundaries have been identified via the map $\{-\pi\} \times \mathbb{R} \rightarrow$ $\{\pi\} \times \mathbb{R}:(-\pi, y) \mapsto(\pi,-y)$.

The identification/extension we just described corresponds to the projective completion of an affine plane as follows: By adding the verticals as lines to the line set of $\left(P_{p}, L_{p}\right)$ we arrive at a so-called affine plane, i.e., an incidence structure satisfying axioms A1 and A2, where A2 = DA2 and A1 results from DA1 by replacing the word "non-parallel" by "distinct". Furthermore, this affine plane is flat or 2dimensional in the sense of Salzmann [21], since its point set is homeomorphic to $\mathbb{R}^{2}$ and all lines are homeomorphic to $\mathbb{R}$ and separate the point set $P_{p}$ into two open components. An example of a flat affine plane everybody is familiar with is the affine plane over the reals. In fact, the "derived" affine planes at all points of the classical flat Laguerre plane over the reals are isomorphic to the affine plane over the reals. The point set of the projective completion of any flat affine plane by its ideal line can be identified with the point set of the projective plane over the reals such that all lines in the plane are homeomorphic to the circle. If we remove the ideal point of the verticals from this topological space we are left with a Möbius strip, and the verticals in the flat dually affine plane we started with plus the ideal line punctured at this ideal point form a set of generators on the Möbius strip $M$. The incidence structure we are looking for is $\left(M, \bar{L}_{a}\right)$, where $\bar{L}_{a}$ consists of all lines that do not pass through the ideal point of the verticals. All these lines are homeomorphic to the circle, and will therefore again be called circles, and every single one of these circles corresponds to exactly one of the lines in the flat dually affine plane $\left(P_{a}, L_{a}\right)$.

Let us call two points on the Möbius strip $M$ parallel if they are both contained in the ideal line or if they correspond to parallel points in the flat dually affine plane. Clearly, the incidence structure $\left(M, \bar{L}_{a}\right)$ satisfies the following axioms:

(M1) Two non-parallel points can be uniquely joined by a circle.

(M2) Two circles intersect in a unique point.

Remember that we want to think of a Möbius strip as the set $[-\pi, \pi] \times \mathbb{R}$ whose left and right boundaries have been identified via the map $\{-\pi\} \times \mathbb{R} \rightarrow\{\pi\} \times \mathbb{R}$ : $(-\pi, y) \mapsto(\pi,-y)$. Since the point set of $\left(M, \bar{L}_{a}\right)$ is a Möbius strip, its circles correspond to (graphs of) continuous half-periodic functions $f:[-\pi, \pi] \rightarrow \mathbb{R}$, i.e., functions such that $f(-\pi)=-f(\pi)$. Because of this and because of M1, it seems natural to call the set of all these functions a half-periodic 2-unisolvent set of functions. We will call any incidence structure $(M, \bar{L})$ a Möbius strip plane if it satisfies axioms M1 and M2 and if $\bar{L}$ consists of graphs of continuous half-periodic functions.

It seems that half-periodic $n$-unisolvent sets and half-periodic Chebyshev systems have been largely ignored in the theory of interpolation, whereas a lot has been written about periodic $n$-unisolvent sets and periodic Chebyshev systems. It is a well-known fact that periodic $n$-unisolvent sets exist only if $n$ is an odd integer. On the other hand, we will show that half-periodic $n$-unisolvent sets exist only if $n$ is an even integer. We also remark that it is possible to prove results about things like best approximation of continuous half-periodic functions by linear or non-linear half-periodic $n$-unisolvent sets that correspond to well-known results about periodic $n$-unisolvent sets. We will not pursue this point any further in this paper.

Back to the issue at hand. We started with a flat Laguerre plane, i.e., essentially a special kind of periodic 3 -unisolvent set, derived at a point by removing the vertical through a point $p$ and concentrating on all the circles through $p$. Finally, we glued 


\section{periodic \\ 1-unisolvent set}

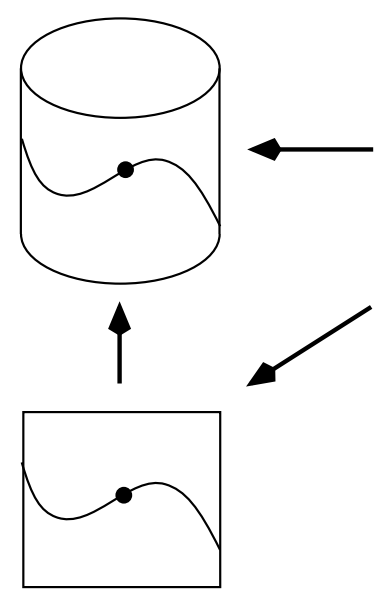

1-unisolvent set


2-unisolvent set

\section{periodic \\ 3-unisolvent set \\ flat Laguerre plane}

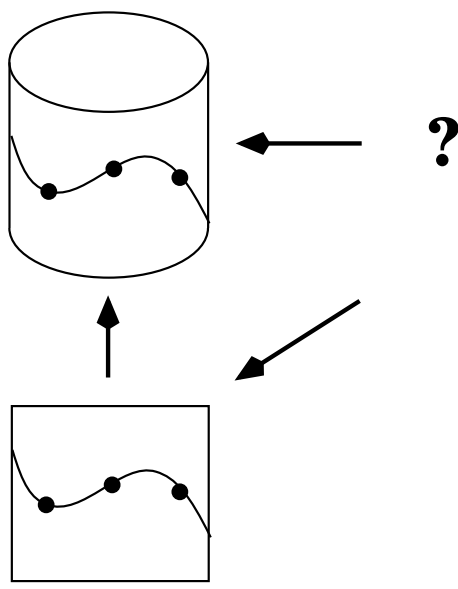

3-unisolvent set

FiguRE 3

another vertical back in to arrive at a Möbius strip plane that is, essentially, a half-periodic 2-unisolvent set. It is easy to see that deriving, in the same way as above, at a point of the Möbius strip plane yields an incidence structure of lines in $(-\pi, \pi) \times \mathbb{R}$ such that every point in this "strip" is contained in exactly one such line. Again, we can identify the right and left boundaries of this strip with a copy of $\mathbb{R}$ and extend all lines by some point in this copy such that the resulting space is homeomorphic to the cylinder, all lines plus their respective added points turn into sets homeomorphic to the circle, and every point on the cylinder is contained in precisely one of these circles. So, essentially, we get a periodic 1-unisolvent set. We arrive at the picture depicted in Figure 3.

A natural question to ask is whether we can extend this diagram to the right. So, for example, does there exist a half-periodic 4-unisolvent set (on a Möbius strip) all of whose derived incidence structures are, essentially, flat Laguerre planes? In Section 4 we will answer this question in the affirmative by constructing nested periodic and half-periodic $n$-unisolvent sets.

For general information about the topics mentioned in this section the reader is referred to: [5], [6], [26] for flat Laguerre planes; [21], [22] for flat affine and projective planes; [1] for Möbius strip planes; [10], [23] for Chebyshev systems; [2], [15], [19], [20], [27] for unisolvent sets.

Notation and conventions. All intervals of $\mathbb{R}$ in this paper contain infinitely many points. (We want to exclude the intervals that contain only one point.)

If $F$ is a set of continuous functions defined on an interval $I$, then the restriction of $F$ to the subinterval $I^{\prime}$ of $I$ is the set of restrictions of functions in $F$ to $I^{\prime}$. If $f \in F$, its restriction to $I^{\prime}$ will be denoted by $f_{I^{\prime}}$. A closed, open, half-open interval 
with left boundary $a$ and right boundary $b$ is denoted by $[a, b],(a, b),[a, b)((a, b])$, respectively.

A periodic function $f$ is a function $[-\pi, \pi] \rightarrow \mathbb{R}$ such that $f(-\pi)=f(\pi)$. A halfperiodic (or antiperiodic) function $f$ is a function $[-\pi, \pi] \rightarrow \mathbb{R}$ such that $f(-\pi)=$ $-f(\pi)$.

\section{PERIODIC AND HALF-PERIODIC $n$-Unisolvent SETS}

Let $n \geq 1$ be an integer, let $I \subset \mathbb{R}$ be an interval and let $F$ be a set of continuous functions $I \rightarrow \mathbb{R}$. Then $F$ is called an $n$-unisolvent set on $I$ if for any set of points $\left(x_{i}, y_{i}\right) \in I \times \mathbb{R}, i=1, \ldots, n$, with $x_{1}<x_{2}<\ldots<x_{n}$ there exists exactly one $f \in F$ that interpolates these points, i.e., $f\left(x_{i}\right)=y_{i}, i=1, \ldots, n$. If $I=[-\pi, \pi]$, then $F$ is called a (half-)periodic $n$-unisolvent set if the restriction of $F$ to the half-open interval $[-\pi, \pi)$ is $n$-unisolvent on $[-\pi, \pi)$ and all $f \in F$ are (half-)periodic.

We already encountered examples of these three kinds of $n$-unisolvent sets in Section 1: periodic 3-unisolvent sets in the guise of flat Laguerre planes, half-periodic 2-unisolvent sets in the guise of Möbius strip planes and periodic 1-unisolvent sets. Further well-known examples are the periodic $(2 n+1)$-unisolvent set

$$
\operatorname{span}\{1, \sin t, \cos t, \sin 2 t, \cos 2 t, \ldots, \sin n t, \cos n t\}
$$

and the $n$-unisolvent set

$$
\operatorname{span}\left\{1, x, x^{2}, \ldots, x^{n-1}\right\}
$$

on any interval. The last two sets are linear in the sense that they are both linear spans of the two sets $\{1, \sin t, \cos t, \sin 2 t, \cos 2 t, \ldots, \sin n t, \cos n t\}$ and $\left\{1, x, x^{2}\right.$, $\left.\ldots, x^{n-1}\right\}$, respectively. These two sets are the two classical examples of (periodic) Chebyshev systems, i.e., sets of functions the spans of which are (periodic) unisolvent sets. The standard reference for Chebyshev systems is [10] (see also [23] for a neat summary of basic facts about these sets).

As seen in the previous section, incidence geometers usually talk about lines or circles instead of functions, points being contained in lines instead of functions interpolating points. So, let us define an incidence structure that is the incidence geometric counterpart of an $n$-unisolvent set.

An $n$-OA (orthogonal array of strength $n)(P, L, \|)$ is an incidence structure consisting of a point set $P$, a line set $L$, all of whose elements are subsets of $P$, and an equivalence relation on the point set (parallelism), such that the following axioms are satisfied:

(O1) Every set of $n$ pairwise non-parallel points is contained in a exactly one line.

(O2) Any line and parallel class intersect in exactly one point.

This definition is a little bit too general for our purposes. So we specify further.

An $n$-OA is flat if either

1) $P=I \times \mathbb{R}$, where $I$ is an interval, two points $\left(x_{1}, y_{1}\right),\left(x_{2}, y_{2}\right) \in P$ are parallel if and only if $x_{1}=x_{2}$, i.e., both points are contained in the same vertical, and all lines are graphs of continuous functions $I \rightarrow \mathbb{R}$. This means that all lines are homeomorphic to $I$; or

2) $P=[-\pi, \pi] \times \mathbb{R}$, where the left and right boundaries of $P$ have been identified by the map $\lambda:\{-\pi\} \times \mathbb{R} \rightarrow\{\pi\} \times \mathbb{R}:(-\pi, y) \mapsto(\pi, y)$. Furthermore all lines are graphs of continuous periodic functions. This means that all lines in $L$ are homeomorphic to the circle, and $P$ is a cylinder. We will therefore speak 
of circles rather than lines. Two points $\left(x_{1}, y_{1}\right),\left(x_{2}, y_{2}\right) \in P$ are parallel if and only if $x_{1}=x_{2}$; or

3) Same as 2). We just replace $\lambda$ by the map $\{-\pi\} \times \mathbb{R} \rightarrow\{\pi\} \times \mathbb{R}:(-\pi, y) \mapsto$ $(\pi,-y)$. In this case $P$ is a Möbius strip. All lines are graphs of continuous half-periodic functions and are therefore homeomorphic to the circle. Again we will speak of circles rather than lines.

Clearly, the flat $n$-OAs in 1) correspond to the $n$-unisolvent sets on $I$. The flat $n$ OAs in 2) correspond to the periodic $n$-unisolvent sets, and $P$ is a cylinder. Finally, the flat $n$-OAs in 3) correspond to the half-periodic $n$-unisolvent sets, and $P$ is a Möbius strip. It is also worth pointing out that the parallel class that corresponds to the identified boundaries in 2) and 3) is in no way distinguished among the parallel classes of these flat $n$-OAs.

Incidence geometers and the mathematicians who first investigated $n$-unisolvent sets, Chebyshev systems and the like all came up with the same definition of when two $n$-unisolvent sets should be considered equivalent; we say that two flat $n$-OAs (or $n$-unisolvent sets) are topologically equivalent if there exists a homeomorphism $\nu$ between the point sets of the (associated) flat $n$-OAs that maps parallel classes to parallel classes and lines, circles to lines, circles, respectively. Incidence geometers refer to the homeomorphism $\nu$ as a continuous isomorphism (or collineation). We remark that all periodic 1-unisolvent sets are topologically equivalent. Also if $I, I^{\prime}$ are intervals, then 1-unisolvent sets on $I$ and $I^{\prime}$ are topologically equivalent if and only if $I$ and $I^{\prime}$ are homeomorphic. Let $r \in[0,2 \pi)$ and let $f$ be a (half-)periodic function. Furthermore, let

$$
f_{\text {double }}:[-3 \pi, \pi] \rightarrow \mathbb{R}: x \mapsto \begin{cases}f(x+2 \pi)(-f(x+2 \pi)) & \text { for } x \in[-3 \pi,-\pi], \\ f(x) & \text { for } x \in[-\pi, \pi] .\end{cases}
$$

Then $f_{r \rightarrow}:[-\pi, \pi] \rightarrow \mathbb{R}: x \mapsto f_{\text {double }}(x-r)$ is also a (half-)periodic function. If $F$ is a set of (half-)periodic functions we call $F_{r \rightarrow}=\left\{f_{r \rightarrow \mid f} \in F\right\}, r \in[0,2 \pi$ ), the $r$-shift of $F$. We observe

Lemma 2.1. Let $F$ be a (half-)periodic $n$-unisolvent set and let $r \in[0,2 \pi)$. Then $F_{r \rightarrow}$ is a (half-)periodic n-unisolvent set that is topologically equivalent to $F$.

Let $O$ be a flat (half-)periodic $n$-OA and let $F$ be its corresponding (half-)periodic $n$-unisolvent set. The (half-)periodic n-unisolvent set that corresponds to $O$ with respect to a point $(r, s) \in[-\pi, \pi) \times \mathbb{R}$ is the $(\pi-r)$-shift of $F$. Notice that in the (half-)periodic $n$-OA that corresponds to the $(\pi-r)$-shift of $F$ the point that corresponds to the point $(r, s)$ has abscissa $\pi$. We see that, indeed, the parallel class $\{-\pi\} \times \mathbb{R}=\{\pi\} \times \mathbb{R}$ is in no way distinguished among the parallel classes of these flat $n$-OAs.

Let $F$ be a (periodic, half-periodic) $n$-unisolvent set on an interval $I([-\pi, \pi])$. Let $f, g \in F, f \neq g$, and let $x_{0} \in I$ be a zero of the function $f-g$. If $x_{0}$ is contained in the interior of $I$ and $f-g$ does not change signs in a suitable small neighborhood of $x_{0}$, then we will say that $x_{0}$ is a non-nodal zero. Otherwise $x_{0}$ is a nodal zero. If we are dealing with an $n$-unisolvent set, every boundary point of $I$ that is a zero is considered as a nodal zero. If we are dealing with a (half-)periodic $n$-unisolvent set, then a zero at $-\pi$ is non-nodal if in some neighborhoods of $-\pi$ and $\pi$ the function $f-g$ has the same (opposite) sign. Otherwise it is a nodal zero. In all these cases nodal zeros have simplified geometric multiplicity 1 and non-nodal zeros have simplified geometric multiplicity 2. 


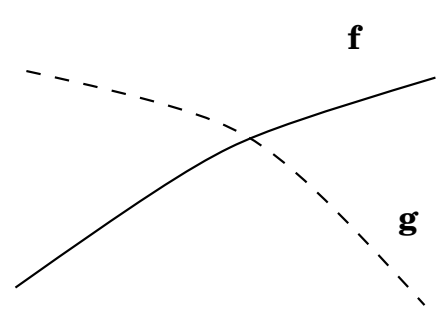

graphs intersect transversally

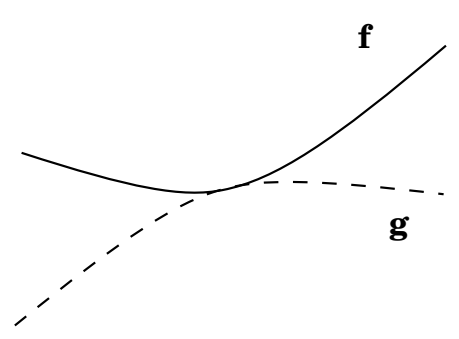

graphs touch locally

FiguRE 4

In the flat (periodic, half-periodic) $n$-OA that corresponds to $F$ the graphs of $f$ and $g$ are (circles) lines of the $n$-OA. If $x_{0}$ is a nodal zero then these graphs intersect transversally at the point $\left(x_{0}, f\left(x_{0}\right)\right)$. Otherwise they touch locally at the point $\left(x_{0}, f\left(x_{0}\right)\right)$ (see Figure 4 ).

If $F$ is an $n$-unisolvent set, let $S G M(f, g)$ denote the sum of the simplified geometric multiplicities of the zeros of $f-g$. If $F$ is a periodic or half-periodic $n$-unisolvent set, let $S G M(f, g)$ denote the sum of the simplified geometric multiplicities of the zeros of $f-g$ that are contained in the interval $[-\pi, \pi)$. Curtis $[2$, Theorem 2] proved the following result for closed intervals.

Proposition 2.2. Let $F$ be an $n$-unisolvent set on the interval $I$ and let $f, g \in F$, $f \neq g$. Then $S G M(f, g) \leq n-1$.

Proof. If $I$ is a general interval and $f$ and $g$ are as in the proposition, then the zeros of $f-g$ are contained in a closed subinterval $I^{\prime}$ of $I$ such that none of these zeros coincides with an interval end of $I^{\prime}$ unless it is an interval end of $I$. Then the restriction of $F$ to $I^{\prime}$ is also an $n$-unisolvent set and $S G M(f, g)=S G M\left(f_{I^{\prime}}, g_{I^{\prime}}\right)$. But $S G M\left(f_{I^{\prime}}, g_{I^{\prime}}\right) \leq n-1$ by Curtis' result [2, Theorem 2].

An easy corollary of this is

Corollary 2.3. Let $F$ be a (half-)periodic $n$-unisolvent set and let $f, g \in F, f \neq g$. Then $\operatorname{SGM}(f, g) \leq n-1$.

Proof. Clearly $S G M\left(f_{r \rightarrow}, g_{r \rightarrow}\right)=S G M(f, g)$ for all $r \in[0,2 \pi)$. Therefore we may assume that all zeros of $f-g$ are contained in $(-\pi, \pi)$. Since the restriction of $F$ to this interval is an $n$-unisolvent set, the corollary follows from the last proposition.

Tornheim [27, Theorem 5] proved part a) of the following proposition. Curtis [2, Theorem 1] proved part b) for periodic $n$-unisolvent sets. His proof also works in the case of half-periodic $n$-unisolvent sets.

Proposition 2.4. a) Let $F$ be an n-unisolvent set on the closed interval $[a, b]$. Let $\left(x_{1}^{(k)}\right)_{k \in \mathbb{N}}, \ldots,\left(x_{n}^{(k)}\right)_{k \in \mathbb{N}},\left(y_{1}^{(k)}\right)_{k \in \mathbb{N}}, \ldots,\left(y_{n}^{(k)}\right)_{k \in \mathbb{N}}$ be $2 n$ sequences of real numbers such that $a \leq x_{1}^{(k)}<\ldots<x_{n}^{(k)} \leq b$ for all $k \in \mathbb{N}$, and let $f_{k}$ be the unique function in $F$ such that $f_{k}\left(x_{i}^{(k)}\right)=y_{i}^{(k)}, i=1, \ldots, n$. Suppose for each $i, \lim _{k \rightarrow \infty} x_{i}^{(k)}=x_{i}$, $\lim _{k \rightarrow \infty} y_{i}^{(k)}=y_{i}$ and $a \leq x_{1}<\ldots<x_{n} \leq b$. Let $f$ be the unique function in $F$ such that $f\left(x_{i}\right)=y_{i}, i=1, \ldots, n$. Then $f=\lim _{k \rightarrow \infty} f_{k}$ uniformly on $[a, b]$. 
b) Let $F$ be a (half-)periodic $n$-unisolvent set. Let $\left(x_{1}^{(k)}\right)_{k \in \mathbb{N}}, \ldots,\left(x_{n}^{(k)}\right)_{k \in \mathbb{N}}$, $\left(y_{1}^{(k)}\right)_{k \in \mathbb{N}}, \ldots,\left(y_{n}^{(k)}\right)_{k \in \mathbb{N}}$ be $2 n$ sequences of real numbers such that $-\pi \leq x_{1}^{(k)}<$ $\ldots<x_{n}^{(k)}<\pi$ for all $k \in \mathbb{N}$, and let $f_{k}$ be the unique function in $F$ such that $f_{k}\left(x_{i}^{(k)}\right)=y_{i}^{(k)}, i=1, \ldots, n$. Suppose for each $i, \lim _{k \rightarrow \infty} x_{i}^{(k)}=x_{i}, \lim _{k \rightarrow \infty} y_{i}^{(k)}=$ $y_{i}$ and $-\pi \leq x_{1}<\ldots<x_{n}<\pi$. Let $f$ be the unique function in $F$ such that $f\left(x_{i}\right)=y_{i}, i=1, \ldots, n$. Then $f=\lim _{k \rightarrow \infty} f_{k}$ uniformly on $[-\pi, \pi]$.

The following result allows us to construct periodic and half-periodic $n$-unisolvent sets from $n$-unisolvent sets.

Proposition 2.5. Let $F$ be an n-unisolvent set, $n \geq 2$, on the interval $[-\pi, \pi]$.

If $n$ is an even integer, let $F^{\prime}=\{f \in F \mid f(-\pi)=f(\pi)\}$. Then $F^{\prime}$ is a periodic $(n-1)$-unisolvent set.

If $n$ is an odd integer, let $F^{\prime}=\{f \in F \mid f(-\pi)=-f(\pi)\}$. Then $F^{\prime}$ is a halfperiodic $(n-1)$-unisolvent set.

Proof. Let $n$ be an even integer. Let $\left(x_{i}, y_{i}\right) \in[-\pi, \pi) \times \mathbb{R}, i=1,2, \ldots, n-1$, be pairwise non-parallel points. We show that there exists a uniquely determined function $f \in F^{\prime}$ that interpolates these points. If the abscissa of one of the points is $-\pi$, then this is clearly true. If this is not the case, let $G$ be the set of all functions in $F$ that interpolate these $n-1$ points. Let $f, g \in G, f \neq g$. Then, by definition, the function $f-g$ has $n-1$ distinct zeros. By Proposition 2.2, these are all the zeros of $f-g$, and all zeros are nodal. This means that $f-g$ changes sign exactly $n-1$ times. Since $n-1$ is an odd integer, we conclude that $f(-\pi) \lessgtr g(-\pi)$ implies $f(\pi) \gtrless g(\pi)$. Let $f_{t}, t \in \mathbb{R}$, be the uniquely determined function in $G$ with $f_{t}(-\pi)=t$. Define a map $\lambda: \mathbb{R} \rightarrow \mathbb{R}: t \mapsto f_{t}(\pi)$. From what we just said it is clear that this map is strictly decreasing and bijective, i.e., it is a strictly decreasing homeomorphism. Every such homeomorphism has exactly one fixed point $t_{0} \in \mathbb{R}$. Hence $f_{t_{0}}(\pi)=t_{0}=f_{t_{0}}(-\pi)$. Hence $f_{t_{0}}$ is the function we have been looking for.

The case where $n$ is odd is dealt with in a similar fashion.

This method of constructing periodic and half-periodic $n$-unisolvent sets has been inspired by an idea of Lorentz [13, Theorem 1], who constructs periodic Chebyshev systems from Chebyshev systems of even order in this manner. Notice that if $F$, above, is a linear set, then $F^{\prime}$ will also be a linear set.

Proposition 2.6. Periodic, half-periodic $n$-unisolvent sets exist if and only if $n$ is odd, even, respectively.

Proof. Since $\operatorname{span}\left\{1, x, x^{2}, \ldots, x^{n-1}\right\}$ is an $n$-unisolvent set on any interval, we can use the last proposition to prove that the "if" part of our assertion is true.

That there are no periodic $n$-unisovent sets if $n$ is even has been proved by Curtis [2, p. 1016, Corollary], using Proposition 2.2. Essentially the same argument can be used to conclude that there are no half-periodic $n$-unisolvent sets if $n$ is odd.

This result suggests coining an " $n$-name" that denotes a periodic, half-periodic set if $n$ is odd, even, respectively. For want of a better idea we choose $n$-phunisolvent as this $n$-name. We will call the flat $n$-OA associated with an $n$-phunisolvent set an $n$-phOA. 
Two more obvious methods of constructing $n$-unisolvent sets are described in the following

Proposition 2.7. a) Let $F$ be an $n$-unisolvent set on the interval $I$ and let $\left(x_{i}, y_{i}\right) \in$ $I \times \mathbb{R}, i=0,1, \ldots, m, m \leq n-1$, be mutually non-parallel points. Let $I^{\prime}$ be a subinterval of $I$ that does not contain any of the $x_{i} s$ and let $F^{\prime}=\left\{f_{I^{\prime}} \mid f \in\right.$ $\left.F, f\left(x_{i}\right)=y_{i}, i=1,2, \ldots, m\right\}$. Then $F^{\prime}$ is an $(n-m)$-unisolvent set on $I^{\prime}$.

b) Let $F$ be an $n$-phunisolvent set, let $y_{0} \in \mathbb{R}$ and let $F^{\prime}=\left\{f_{(-\pi, \pi)} \mid f \in F, f(\pi)=\right.$ $\left.y_{0}\right\}$. Then $F^{\prime}$ is an $(n-1)$-unisolvent set on $(-\pi, \pi)$.

2.8. Remarks. 1) It is mainly combinatorists and incidence geometers who are interested in $n$-OAs, especially $n$-OAs that are defined on finite point sets. See [4] for general information about finite $n$-OAs.

2) A general (not necessarily flat) $n$-OA $(P, L, \|)$ is maximal if it cannot be extended to a bigger $n$-OA by attaching a parallel class of points to $P$ and extending every line in $L$ by exactly one point in this parallel class. The $n$-OA that corresponds to $\operatorname{span}\left\{1, x, \ldots, x^{n-1}\right\}$ defined on $[0,1]$ is not maximal, since we can extend this $n$-OA to a bigger $n$-OA by attaching the vertical through the point $(2,0)$ to it and extending all its lines in the natural way. It is, in general, very difficult to decide whether a given $n$-OA is maximal or not. Therefore the following corollary of Proposition 2.6 comes as a pleasant surprise.

Proposition 2.8.1. An $n-p h O A, n \geq 2$, is a maximal $n-O A$.

Proof. Let us assume that $O$ is a flat $n$-OA on the cylinder and that $\bar{O}$ is an extension of this $n$-OA by some parallel class to a "bigger" $n$-OA. Then the set of all circles in $O$ that get extended by a certain fixed point in this parallel class is a circle set of a flat $(n-1)$-OA on the cylinder. This contradicts Proposition 2.6. The case where $O$ is a flat $n$-OA on the Möbius strip is dealt with in a similar manner.

Clearly, there do not exist any maximal 1-OAs.

3) We want to remark that the periodic $n$-OAs that correspond to linear $n$ unisolvent sets of the form $\operatorname{span}\left\{1, u_{1}(t), u_{2}(t), \ldots, u_{n-1}(t)\right\}$ can be represented on a cylinder in $\mathbb{R}^{n}$ very much like the flat Laguerre planes we considered in the first section. Let $K$ be the graph of the function $[-\pi, \pi] \rightarrow \mathbb{R}^{n-1}: t \mapsto$ $\left(u_{1}(t), u_{2}(t), \ldots, u_{n-1}(t)\right)$. Then $K$ can be shown to be a maximal $(n-1)$-arc in $\mathbb{R}^{n-1}$, i.e., a curve that is intersected by any hyperplane of $\mathbb{R}^{n-1}$ in no more than $n-1$ points. Furthermore this curve is closed and does not intersect itself. For $n=3$ this is the same as saying that $K$ is a strictly convex simple closed curve. The cylinder $Z$ over $K$ in $\mathbb{R}^{n}$ is the set $\left\{\left(x_{1}, x_{2}, \ldots, x_{n-1}, x_{n}\right) \mid\left(x_{1}, x_{2}, \ldots, x_{n-1}\right) \in K, x_{n} \in\right.$ $\mathbb{R}\}$. The verticals on this cylinder are the sets $\left\{\left(x_{1}, x_{2}, \ldots, x_{n-1}, x_{n}\right) \mid x_{n} \in \mathbb{R}\right\}$, $\left(x_{1}, x_{2}, \ldots, x_{n-1}\right) \in K$. Two points on $Z$ are parallel if they are contained in the same vertical. Let $C$ be the set of intersections of the non-vertical hyperplanes of $\mathbb{R}^{n}$ with the cylinder. Then $(Z, C, \|)$ is the flat $n$-OA on the cylinder associated with the periodic $n$-unisolvent set we started out with. In this context see also the discussion of moment spaces of periodic Chebyshev systems in [10, Chapter 2 and Chapter 6]. We also want to mention that in $\mathbb{R}^{m}$, where $m$ is an odd integer, there exist no maximal $m$-arcs that are simply closed curves. As above, such an arc would give rise to a $(m+1)$-phOA on the cylinder. By Proposition 2.6, this is impossible. 


\section{Nested $n$-(PH) UnisOlvent SETS AND NESTED $n$-(PH)OAS}

We already mentioned at the end of Section 1 that we are interested in constructing $n$-phOAs that are generalizations of flat Laguerre planes und Möbius strip planes, i.e., we want to extend Figure 3 to the right.

Let $(P, C, \|)$ be an $n$-phOA, $n>1$, and let $p$ be a point in $P$. The restricted $n$-OA at the point $p$ is $\left(P_{p}^{r e s}, C_{p}^{r e s}, \|\right)$ where $P_{p}^{r e s}$ is $P$ minus the parallel class $p$ is contained in and $C_{p}^{r e s}$ consists of all circles in $C$ that have been punctured at this parallel class. We note that the restricted $n$-OAs at parallel points coincide. The derived $(n-1)$-OA at the point $p$ is $\left(P_{p}^{d e r}, C_{p}^{d e r}, \|\right)$, where $P_{p}^{d e r}=P_{p}^{r e s}$ and $C_{p}^{d e r}$ consists of all circles in $C$ through $p$ that have been punctured at $p$. Let $F$ be the $n$-phunisolvent set associated with $(P, C, \|)$ and let $E$ be the $n$-phunisolvent set associated with $(P, C, \|)$ with respect to $p$ (see the definition following Lemma 2.1). The restriction of the subset of $E$ that corresponds to $\left(P_{p}^{r e s}, C_{p}^{r e s}, \|\right)$ to the interval $(-\pi, \pi)$ is the restricted $n$-unisolvent set of $F$ at the point $p$. Similarly, the restriction of the subset of $E$ that corresponds to $\left(P_{p}^{d e r}, C_{p}^{d e r}, \|\right)$ to the interval $(-\pi, \pi)$ is the derived $(n-1)$-unisolvent set of $F$ at the point $p$.

Let $F$ be an $n$-phunisolvent set and let $O$ be its associated $n$-phOA. Then $F$ and $O$ are nested if and only if:

$n=1$ : automatically;

$n>1$ : for every $p \in P$ there exists a nested $(n-1)$-phOA $O_{p}$ whose restricted $(n-1)-\mathrm{OA}$ at some point is topologically equivalent to the derived $(n-1)$ $\mathrm{OA}$ of $O$ at the point $p$.

Remember that nested $n$-phOAs are supposed to be generalizations of the flat Laguerre planes and the Möbius strip planes that we discussed in Section 1. In Section 1 we found that for every point $p$ in a flat Laguerre plane there exists a 2phOA (a Möbius strip plane) such that the restricted 2-OA of this 2-phOA at some point is topologically equivalent to the derived 2-OA of the flat Laguerre plane at $p$. The following result shows that every Möbius strip plane is a nested 2-phOA. This implies that the flat Laguerre plane we started with is indeed a nested 3-phOA.

Proposition 3.1. Let $O$ be an incidence structure defined on the Möbius strip. Then the following are equivalent: 1) $O$ is a 2-phOA;2) $O$ is a nested 2-phOA; 3) $O$ is a Möbius strip plane.

Proof. Clearly, both 2) and 3) imply 1). Let $O$ be a 2-phOA and let $p$ be a point of the Möbius strip. The derived 1-OA of $O$ at this point is a flat 1-OA with point set $(-\pi, \pi) \times \mathbb{R}$. So is the restricted 1 -OA at any point of any 1 -phOA. We noted already that all flat 1-OAs that share the same point set are topologically equivalent. Hence 1 ) implies 2). Since $O$ is a 2-phOA (on the Möbius strip), two circles in $O$ cannot intersect in more than one point. On the other hand, two circles in $O$ correspond to two different half-periodic continuous functions $f, g:[-\pi, \pi] \rightarrow \mathbb{R}$. For such functions $f(-\pi) \lesseqgtr g(-\pi)$ implies $f(\pi) \gtreqless g(\pi)$. The intermediate value theorem guarantees the existence of an $x_{0} \in[-\pi, \pi)$ such that $f\left(x_{0}\right)=g\left(x_{0}\right)$. Hence any two circles in $O$ intersect in precisely one point. Therefore $O$ is a Möbius strip plane, and 1) implies 3).

3.2. Remark. Keeping in mind the construction of the Möbius strip planes as described in Section 1, we can use Proposition 3.1 to prove [21, Theorem 2.5], which says: Let $P$ be a non-orientable compact surface of genus 1 and let $L$ be a system 
of simple closed curves in $P$. Assume that there is a unique line in $L$ joining each pair of distinct points. Then $(P, L)$ is a flat projective plane.

Another way of putting one of the arguments in the last proof is to say that the derived 1-OA of any nested 2-phOA at a point can be extended in an essentially unique way to a 1 -phOA. Is the respective statement true for $n$-phOAs, $n \geq 3$, i.e., does it make sense to speak of something like THE derived $(n-1)$-phOA of a nested $n$-phOA at some point? The following proposition answers this question in the affirmative.

Proposition 3.3. Let $p h O A_{1}=(P, C, \|)$ and phOA $A_{2}=(P, \bar{C}, \|)$ be two n-phOAs, $n \geq 2$. If $O A_{1}=\left(P_{p}^{r e s}, C_{p}^{r e s}, \|\right)$ and $O A_{2}=\left(P_{p}^{r e s}, \bar{C}_{p}^{r e s}, \|\right)$, where $p \in P$, are topologically equivalent $n-O A s$, then $p h O A_{1}$ and $p h O A_{2}$ are topologically equivalent.

Proof. We may assume that $p$ is one of the points with abscissa $\pi$. Then $O A_{1}$ and $O A_{2}$ are flat $n$-OAs on $(-\pi, \pi) \times \mathbb{R}$.

We distinguish two cases. Let $n$ be an odd integer. Let $F_{1}$ and $F_{2}$ be the periodic $n$-unisolvent sets that correspond to $p h O A_{1}$ and $p h O A_{2}$, respectively. Let $F$ be the $n$-unisolvent set on $(-\pi, \pi)$ that corresponds to $O A_{1}$ and let $\lambda$ be a homeomorphism of $(-\pi, \pi) \times \mathbb{R}$ that establishes the topological equivalence of $O A_{1}$ and $O A_{2}$, i.e., " $\lambda\left(O A_{1}\right)=O A_{2}$ ".

We define two maps $\phi_{i}: F \rightarrow \mathbb{R}, i=1,2$, as follows. Let $f \in F$ and let $f_{1}$ be the uniquely determined function in $F_{1}$ such that the restriction of $f_{1}$ to $(-\pi, \pi)$ is $f$. Then $\phi_{1}(f)=f_{1}(\pi)$. Let $f_{2}$ be the uniquely determined function in $F_{2}$ the graph of whose restriction to $(-\pi, \pi)$ coincides with the image of the graph of $f$ under the homeomorphism $\lambda$. Then $\phi_{2}(f)=f_{2}(\pi)$. The two maps $\phi_{1}, \phi_{2}$ will help us keep track of which functions in $O A_{1}$ and $O A_{2}$ get extended by common points in $p h O A_{1}$ and $p h O A_{2}$, respectively. Let $F_{1 a}, F_{2 a}, a \in \mathbb{R}$, be the set of all functions $f \in F$ such that $\phi_{1}(f)=a, \phi_{2}(f)=a$, respectively.

Let $f, g \in F, f \neq g$. Since $f-g$ has at most $n-1$ zeros, we have a natural "left" and "right" ordering of $F$, i.e., there exists an $\epsilon>0$ such that the signs of $f-g$ stay unchanged in $(-\pi,-\pi+\epsilon)$ and $(\pi-\epsilon, \pi)$. If these pairs of signs are ++ , ,,+--+-- , then we express this by writing $f>>g, f><g, f<>g, f<<g$, respectively.

Now, given any $F_{1 a}$, we know that for all $g \in F \backslash F_{1 a}$ either $f<<g$ for all $f \in F_{1 a}$, or $f>>g$ for all $f \in F_{1 a}$. The respective statement is true for any $F_{2 a}$ since the homeomorphism $\lambda$ preserves (or inverts) the left and right ordering of the elements in $F$. Hence given any $F_{2 a}$, we know that for all $g \in F \backslash F_{2 a}$ either $f<<g$ for all $f \in F_{2 a}$, or $f>>g$ for all $f \in F_{2 a}$. If there exists a bijection $\sigma: \mathbb{R} \rightarrow \mathbb{R}$ such that $f \in F_{1 a}$ if and only if $f \in F_{2 \sigma(a)}$, then this bijection is necessarily strictly increasing or strictly decreasing, i.e., it is a homeomorphism. Moreover, the homeomorphism $\lambda$, which, we say it again, preserves the relative left and right orderings of $F$, extends in a natural way to a homeomorphism $\bar{\lambda}$ of the common point set $P$ of $p h O A_{1}$ and $p h O A_{2}$ that fixes all parallel classes and maps circles to circles, showing that both phOAs are topologically equivalent. This homeomorphism then looks as follows:

$$
\bar{\lambda}:[-\pi, \pi] \times \mathbb{R} \rightarrow[-\pi, \pi] \times \mathbb{R}:(x, y) \mapsto \begin{cases}( \pm \pi, \sigma(y)) & \text { for } x= \pm \pi \\ \lambda(x, y) & \text { otherwise. }\end{cases}
$$

Let $a^{\prime}, b^{\prime} \in \mathbb{R}$. Since both $F_{1 a^{\prime}}$ and $F_{2 b^{\prime}}$ are $(n-1)$-unisolvent on $(-\pi, \pi)$, one cannot be properly contained in the other. So, if there does not exist a bijection $\sigma$ 
as described above, there exist $a, b, c, d \in \mathbb{R}, a<b<c$, and $f, g \in F_{2 d}$ such that $f \in F_{1 a}, g \in F_{1 c}$. If $h \in F_{1 b}$, then $f<<h<<g$ or $f>>h>>g$. If $h$ were not contained in $F_{2 d}$, then $h<<f, g$ or $h>>f, g$. This is not the case. Hence $F_{1 b}$ is properly contained in $F_{2 d}$. A contradiction.

The case where $n$ is an even integer is dealt with in a similar manner.

The following result is an immediate consequence of the above proposition and the definition of nested $n$-phOAs.

Corollary 3.4. Let $O=(P, C, \|)$ be an $n-p h O A, n \geq 2$, let $p$ be a point in $P$ and let $O^{\prime}=\left(P^{\prime}, C^{\prime}, \|\right)$ be an $(n-1)-p h O A$ whose restriction at some point $q$ is topologically equivalent to the derivation of $O$ at $p$. Then $O^{\prime}$ is uniquely determined up to topological equivalence. Furthermore, if $O$ is nested, $O^{\prime}$ is nested, too.

We will call the $(n-1)$-phOA $O^{\prime}$ the derived $(n-1)$-phOA of $O$ at the point $p$.

The following result together with Proposition 3.1 shows that nested $n$-phOAs are really what we are looking for.

Proposition 3.5. Let $O$ be an incidence structure defined on the cylinder. Then the following are equivalent: 1) $O$ is a nested 3-phOA; 2) $O$ is a flat Laguerre plane.

Proof. It follows from our discussion in Section 1 that 2) implies 1). Let $O$ be a nested 3-phOA. Then it is clear from Proposition 3.1 that the derived 2-phOA at a point $p$ is a Möbius strip plane. This implies that the derived 2-OA of $O$ at $p$ is a flat dually affine plane. This in turn implies (cf., for example, $[6,3.10]$ ) that $O$ is a flat Laguerre plane.

If we are given an $n$-phOA and cut out two different parallel classes, we get two halves. In general, there are many different ways to fit these two halves back together to form an $n$-phOA. This means that there are many topologically inequivalent $n$-phOAs which, when we cut them in this way, yield the same (or topologically equivalent) two halves. In [25], e.g., Steinke classifies the topologically inequivalent flat Laguerre planes that can be constructed by fitting together two halves of the classical flat Laguerre plane over the reals, whose construction we described in Section 1. All these constructions have one thing in common, namely that if we cut an $n$-phOA into two halves and glue those two halves back together along the same sides to get an $n$-phOA that is topologically inequivalent to the one we started with, then there are at least some circles whose two halves come from different circles in the original $n$-phOA.

Also, if one cuts some flat $n$-OA along one of its parallel classes, one is left with two parts. Again these two parts can usually be fitted together, along the same two sides, in many different ways to yield topologically inequivalent flat $n$-OAs. The Moulton planes, for example, are flat affine planes, i.e., essentially flat 2-OAs, that are constructed by gluing together two halves of the real affine plane. They are the earliest and probably most famous examples of non-desarguesian affine planes. Of course, these affine planes correspond to Möbius strip planes that arise from the classical Möbius strip plane (see Remark 4.5 a) for a description of this plane) by cutting this plane along two parallel classes and gluing the resulting two halves back together again in a different way. See [16], [21], [24] for general information about these affine planes. Nevertheless, if we insist on gluing the two halves back together again (along the same two sides) into a flat $n$-OA such that corresponding curves on the left and right sides are extended by common points, the resulting 
flat $n$-OA is going to be topologically equivalent to the $n$-OA we started with. The proof of this corresponds to the proof of Proposition 3.3.

For the sake of completeness let us give a definition of nested flat n-OAs. Let $(P, L, \|)$ be a flat $n$-OA, $n>1$, with corresponding $n$-unisolvent set $F$ on $I$. Let $p=\left(x_{0}, y_{0}\right) \in P$. If $x_{0}$ is an inner point of $I$, then the left (right) restricted $n$ unisolvent set of $F$ at $p$ is the restriction of $F$ to the interval $\left\{x \in I \mid x<x_{0}\left(x>x_{0}\right)\right\}$. The derived left (right) $(n-1)$-unisolvent set of $F$ at $p$ is the set of functions $\left\{f \in F \mid f\left(x_{0}\right)=y_{0}\right\}$ restricted to the interval $\left\{x \in I \mid x<x_{0}\left(x>x_{0}\right)\right\}$. If $x_{0}$ is a boundary point of $I$, then the restricted $n$-unisolvent set of $F$ at $p$ is the restriction of $F$ to the interval $I \backslash\left\{x_{0}\right\}$. The derived $(n-1)$-unisolvent set of $F$ at $p$ is the set of functions $\left\{f \in F \mid f\left(x_{0}\right)=y_{0}\right\}$ restricted to the interval $I \backslash\left\{x_{0}\right\}$. The (left or right) restricted $n$-OA of $(P, L, \|)$ at the point $p$ is the flat $n$-OA that corresponds to the (left or right) restricted $n$-unisolvent set of $F$ at $p$. The (left or right) derived $n$-OA of $(P, L, \|)$ at the point $p$ is the flat $n$-OA that corresponds to the (left or right) derived $n$-unisolvent set of $F$ at $p$. A pair of corresponding lines in the left and right derived $(n-1)$-unisolvent sets (or restricted $n$-unisolvent sets) at a point of $(P, L, \|)$ is a pair of lines that comes from one line in $L$. Every line in a flat $n$-OA over an interval has two ends. We call the end of a line in the (right or left) restricted $n$-OA or derived $(n-1)$-OA at $p$ the $c u t$ end if it "ends" in the parallel class of $p$.

A flat $n$-OA $(P, L, \|)$ is nested if and only if:

$n=1$ : automatically;

$n>1$ : for every $p \in P$ there exists a nested flat $(n-1)$-OA $\left(P, L^{\prime}, \|\right)$ whose (left and right) restricted $(n-1)-\mathrm{OA}(\mathrm{s})$ at some point (are) is topologically equivalent to the (left and right) derived $(n-1)-\mathrm{OA}(\mathrm{s})$ of $(P, L, \|)$ at the point $p$. Furthermore, the homeomorphisms that establish the topological equivalences have to map pairs of corresponding lines to pairs of corresponding lines and cut ends to cut ends.

As in the case of $n$-phOAs, it makes perfect sense to speak of $\left(P, L^{\prime}, \|\right)$ as THE derived flat $(n-1)$-OA at the point. As we mentioned before, the proof of this fact runs along the same lines as the proof or Proposition 3.3. In the sequel we will use the expression "the derived flat $(n-1)-\mathrm{OA}$ " only in this (new) sense.

\section{UNRESTRICTED $n$-(PH)UNISOLVENT SETS. EXAMPLES OF NESTED $n$-(PH)OAS}

The aim of this section is to show that the $n$-(ph)OAs associated with unrestricted $n$-(ph)unisolvent sets are nested. We will also see that deriving in our sense has a lot to do with deriving in the analytical sense.

A (half-)periodic function $f:[-\pi, \pi] \rightarrow \mathbb{R}$ is continuously differentiable if its restriction to $(-\pi, \pi)$ is differentiable, the left and right derivatives at $-\pi$ and $\pi$, respectively, exist, and the derivative of $f$ is a continuous (half-)periodic function.

Let $I$ be an interval. An SIC (set of initial conditions) $s$ of order $m \in \mathbb{N}$ on $I$ is an ordered triple $(X, \Lambda, Y)$, where $X=\left\{x_{1}, x_{2}, \ldots, x_{k}\right\}$ is a set of distinct points in $I, \Lambda=\left\{\lambda_{x_{1}}, \lambda_{x_{2}}, \ldots, \lambda_{x_{k}}\right\}$ is a set of positive integers such that $\lambda_{x_{1}}+\lambda_{x_{2}}+$ $\ldots+\lambda_{x_{k}}=m$, and $Y=\left\{Y_{x_{1}}, Y_{x_{2}}, \ldots, Y_{x_{k}}\right\}$, where $Y_{x_{i}}=\left\{y_{x_{i}}^{(0)}, y_{x_{i}}^{(1)}, \ldots, y_{x_{i}}^{\left(\lambda_{x_{i}}-1\right)}\right\}$, $i=1,2, \ldots, k$, is a set of real numbers. We abbreviate all this by writing $s=$ 


$$
\begin{gathered}
\left\{x_{1}, \ldots, x_{k}\left|\lambda_{x_{1}}, \ldots, \lambda_{x_{k}}\right| y_{x_{1}}^{(0)}, \ldots, y_{x_{k}}^{\left(\lambda_{x_{k}}-1\right)}\right\} . \text { A function } f: I \rightarrow \mathbb{R} \text { satisfies } s \text { if } \\
f^{(j)}\left(x_{i}\right)=y_{x_{i}}^{(j)}, \quad i=1,2, \ldots, k, \quad j=0,1, \ldots, \lambda_{x_{i}}-1 .
\end{gathered}
$$

Here $f^{(0)}=f$ and $f^{(j)}, j>1$, denotes the $j$ th derivative of $f$.

If

$$
s=\left\{x_{1}, \ldots, x_{k}\left|\lambda_{x_{1}}, \ldots, \lambda_{x_{k}}\right| y_{x_{1}}^{(0)}, \ldots, y_{x_{k}}^{\left(\lambda_{x_{k}}-1\right)}\right\}
$$

and

$$
\bar{s}=\left\{\bar{x}_{1}, \ldots, \bar{x}_{\bar{k}}\left|\bar{\lambda}_{\bar{x}_{1}}, \ldots, \bar{\lambda}_{\bar{x}_{\bar{k}}}\right| \bar{y}_{\bar{x}_{1}}^{(0)}, \ldots, \bar{y}_{\bar{x}_{\bar{k}}}^{\left(\lambda_{\overline{\bar{x}}}^{\overline{\bar{x}_{\bar{k}}}}-1\right)}\right\}
$$

are two SICs on $I$ of orders $m$ and $\bar{m}$, respectively, then $s$ is contained in $\bar{s}$ if and only if $s$ is "contained" in the natural way in $\bar{s}$, i.e., if and only if $m \leq \bar{m}$ and $\left\{x_{1}, \ldots, x_{k}\right\} \subseteq\left\{\bar{x}_{1}, \ldots, \bar{x}_{\bar{k}}\right\}$ and $\lambda_{x_{i}} \leq \bar{\lambda}_{x_{i}}, i=1, \ldots, k$, and $y_{x_{i}}^{(j)}=\bar{y}_{x_{i}}^{(j)}$, $i=1,2, \ldots, k, j=0,1, \ldots, \lambda_{x_{i}}-1$.

Following Hartman [7], we call a set $F$ of $n-1$ times continuously differentiable functions on $I$ an unrestricted $n$-unisolvent set if for every SIC $s$ of order $n$ on $I$ there exists exactly one $f \in F$ satisfying $s$. Furthermore, we will say that a set $F$ of $n-1$ times continuously differentiable (half-)periodic functions on $[-\pi, \pi]$ is an unrestricted $n$-phunisolvent set if its restriction to $[-\pi, \pi)$ is an unrestricted $n$-unisolvent set. All SICs for $n$-phunisolvent sets will be defined on $[-\pi, \pi)$. Notice that an unrestricted $n$-(ph)unisolvent set, is automatically an $n$-(ph)unisolvent set, since a function satisfying a SIC of the form $\left\{x_{1}, \ldots, x_{n}|1,1, \ldots, 1| y_{x_{1}}^{(0)}, y_{x_{2}}^{(0)}, \ldots, y_{x_{n}}^{(0)}\right\}$ interpolates the $n$ points $\left(x_{1}, y_{x_{1}}^{(0)}\right),\left(x_{2}, y_{x_{2}}^{(0)}\right), \ldots,\left(x_{n}, y_{x_{n}}^{(0)}\right)$. For $F$ to be an unrestricted $n$-(ph)unisolvent set just means that it solves the Hermite interpolation problem [11].

The classical example of an unrestricted $n$-unisolvent set is $\operatorname{span}\left\{1, x, x^{2}, \ldots\right.$, $\left.x^{n-1}\right\}$. This set is linear. Bases of linear (periodic) unrestricted $n$-unisolvent sets are usually called extended Chebyshev systems, cf. [10, p. 6], [7, Section 10].

Only very few examples of (linear) unrestricted periodic $n$-unisolvent sets are known. These are

\section{Examples 4.1.}

1. $\operatorname{span}\left\{1, \sin t, \cos t, \sin 2 t, \cos 2 t, \ldots, \sin \frac{n-1}{2} t, \cos \frac{n-1}{2} t\right\}$, where $n$ is an odd integer; see [9].

2. $\mathcal{P}_{m, r}=\left\{P \in \Pi_{m} \mid P^{(i)}(-\pi)=P^{(i)}(\pi), i=0,1, \ldots, r\right\}$, where $n=m-r$ is an odd integer and $\Pi_{m}$ is the set of all polynomials of degree at most $m$. These sets are all linear and have been discovered by Lorentz [12, p. 23], [13, p. 19].

3. the Chebyshev spaces generated by a particular Krein kernel as given by Forst [3].

We have the following obvious

Lemma 4.2. Let $F$ be a set of $n-1$ times continuously differentiable (half-)periodic functions and let $r \in[0,2 \pi)$. Then $F$ is an unrestricted n-phunisolvent set if and only if its $r$-shift $F_{r \rightarrow}$ is such a set.

An $n$-(ph)unisolvent set $F$ on the interval $I([-\pi, \pi])$, all of whose elements are $n-1$ times continuously differentiable, is said to have the property of unique $n$ initial values if all SICs of the form $\left\{x_{1}|n| y_{x_{1}}^{(0)}, y_{x_{1}}^{(1)}, \ldots, y_{x_{1}}^{(n-1)}\right\}$ on $I([-\pi, \pi))$ are satisfied by exactly one $f \in F$. Hartman proved [7] 
Proposition 4.3. A set of $n-1$ times continuously differentiable functions on an open interval is an unrestricted $n$-unisolvent set if and only if it is an $n$-unisolvent set and has the property of unique $n$ initial values.

Mathsen [14] showed that this result is false if we replace "open interval" by "closed interval". It is still undecided whether the result is true if we replace "open interval" by "half-open interval". Nevertheless one can show

Corollary 4.4. An n-phunisolvent set $F$ all of whose elements are $n-1$ times continuously differentiable is unrestricted if and only if it has the property of unique $n$ initial values.

Proof. By definition, unrestricted $n$-phunisolvent sets satisfy the property of unique $n$ initial values. On the other hand, let $F$ be an $n$-phunisolvent set $F$ all of whose elements are $n-1$ times continuously differentiable and let $s$ be an SIC on $[-\pi, \pi)$ of order $n$. As a consequence of Lemma 4.2, we may assume that $s$ is an SIC on the open interval $(-\pi, \pi)$. The restriction $F^{\prime}$ of $F$ to $(-\pi, \pi)$ is an unrestricted $n$-unisolvent set. So, there exists exactly one function $f^{\prime}$ in $F^{\prime}$ that satisfies $s$. Clearly, the function $f \in F$ whose restriction to $(-\pi, \pi)$ is $f^{\prime}$ also satisfies $s$ and is the only such element of $F$.

4.5. Remarks. a) Example 4.1.1 is the classical example of an unrestricted periodic $n$-unisolvent set. We have not been able to find the corresponding "classical" example of an unrestricted half-periodic $n$-unisolvent set anywhere in the literature. Here it is:

4.5.1. The classical half-periodic unrestricted $n$-unisolvent set. The set $\operatorname{span}\left\{\sin \frac{t}{2}, \cos \frac{t}{2}, \sin 3 \frac{t}{2}, \cos 3 \frac{t}{2}, \sin 5 \frac{t}{2}, \cos 5 \frac{t}{2}, \ldots, \sin (2 k-1) \frac{t}{2}, \cos (2 k-1) \frac{t}{2}\right\}$ is an unrestricted half-periodic ( $2 k)$-unisolvent set.

Proof. Clearly, the set under consideration is a set of infinitely often continuously differentiable half-periodic functions.

Let $B=\{\sin t, \cos t, \sin 3 t, \cos 3 t, \sin 5 t, \cos 5 t \ldots, \sin (2 k-1) t, \cos (2 k-1) t\}$, let $\bar{B}=\{1, \sin t, \cos t, \sin 2 t, \cos 2 t, \sin 3 t, \cos 3 t, \ldots, \sin (2 k-1) t, \cos (2 k-1) t\}$, let $F=$ $\operatorname{span} B$ and let $\bar{F}=\operatorname{span} \bar{B}$. Now it is a well-known fact (cf. Example 4.1.1) that $\bar{F}$ is an unrestricted $(4 k-1)$-unisolvent set on any subinterval of $[-\pi, \pi)$ and a $(4 k-1)$ dimensional real vector space with basis $\bar{B}$. We show that $F$ is a $(2 k)$-unisolvent set on $[0, \pi)$. Let $x_{1}, x_{2}, \ldots, x_{2 k}$ be distinct points in $[0, \pi)$ and let $y_{1}, y_{2}, \ldots, y_{2 k}$ be real numbers. Let $f_{i}, i=1,2, \ldots 2 k$ be the uniquely determined function in $\bar{F}$ such that $f_{i}\left(x_{j}\right)=-f_{i}\left(x_{j}-\pi\right)=\left\{\begin{array}{ll}0 & \text { if } i \neq j \\ 1 & \text { if } i=j\end{array}\right.$ for $j=1,2, \ldots, 2 k-1$ and $f_{i}\left(x_{2 k}\right)=\left\{\begin{array}{ll}0 & \text { if } 2 k \neq i \\ 1 & \text { if } 2 k=i\end{array}\right.$. Then $G=\operatorname{span}\left\{f_{1}, f_{2}, \ldots, f_{2 k}\right\}$ is the set of all functions $f \in \bar{F}$ such that $f\left(x_{j}\right)=-f\left(x_{j}-\pi\right), j=1,2, \ldots, 2 k-1$. Clearly, $F$ is contained in $G$, and since $G$ has dimension at most $2 k$ we have $F=G$. This shows that $F$ is $(2 k)$-unisolvent on $[0, \pi)$.

We show that $F$ has the property of unique $2 k$ initial values in exactly the same way. Let $x_{0} \in[0, \pi)$ and let $h_{i}, i=0,1, \ldots, 2 k-1$, be the unique function in $\bar{F}$ that has the property $h_{i}^{(j)}\left(x_{0}\right)=-h_{i}^{(j)}\left(x_{0}-\pi\right)=\left\{\begin{array}{ll}0 & \text { if } i \neq j \\ 1 & \text { if } i=j\end{array}\right.$ for $j=0,1, \ldots, 2 k-2$ 
and $h_{i}^{(2 k-1)}\left(x_{0}\right)=\left\{\begin{array}{ll}0 & \text { if } 2 k-1 \neq i \\ 1 & \text { if } 2 k-1=i\end{array}\right.$. Then $H=\operatorname{span}\left\{h_{0}, h_{1}, \ldots, h_{2 k-1}\right\}$ is the set of all $h \in \bar{F}$ with the property $h^{(j)}\left(x_{0}\right)=-h^{(j)}\left(x_{0}-\pi\right)(j=0,1, \ldots, 2 k-2)$. Clearly, $F$ is contained in $H$, and since $H$ has dimension at most $2 k$ we have $F=H$. This shows that $F$ has the property of unique $2 k$ initial values in $[0, \pi)$. We rescale $F$ in a linear fashion and arrive at the set of half-periodic functions under discussion. Clearly, this set is also $(2 k)$-unisolvent on $[-\pi,-\pi)$ and has the property of unique $2 k$ initial values. By Corollary 4.4, it is an unrestricted $n$-phunisolvent set.

It is not difficult to show that the flat projective plane that corresponds to the half-periodic 2-unisolvent set $\operatorname{span}\left\{\sin \frac{t}{2}, \cos \frac{t}{2}\right\}$ is the classical projective plane over the reals.

b) The construction of unrestricted periodic $n$-unisolvent sets mentioned in Example 4.1.2 can be generalized as follows: Let $n=m-r \geq 1$ and let $\Pi_{m}$ be the set of all polynomials of degree at most $m$. Then $\mathcal{P}_{m, r}=\left\{P \in \Pi_{m} \mid P^{(i)}(-\pi)=\right.$ $\left.(-1)^{n+1} P^{(i)}(\pi), i=0,1, \ldots, r\right\}$ is an unrestricted $n$-phunisolvent set. The proof of this fact can be obtained by slightly modifying the proof of Theorem 10 and Lemma 11 in [12].

Maybe this construction can be generalized even further by replacing $\Pi_{m}$ by other unrestricted $(m+1)$-unisolvent sets.

Let $F$ be an unrestricted $n$-unisolvent set on the open interval $I$ or an unrestricted $n$-phunisolvent set. Let $f, g \in F, f \neq g$, and let $x_{0} \in I$ (or $x_{0} \in[-\pi, \pi)$ ) be a zero of $f-g$. Then this zero is of analytic multiplicity $m \in \mathbb{N}$ if $(f-g)^{(j)}\left(x_{0}\right)=0$, $j=0,1, \ldots, m-1$, and $(f-g)^{(m)}\left(x_{0}\right) \neq 0$. Proposition 4.6, below, guarantees that $(f-g)^{(j)}\left(x_{0}\right)=0$ for all $j=0,1, \ldots, n-1$ implies that $f=g$. Therefore analytic multiplicity is well-defined (remember that, for all we know, the elements of $F$ are only $n-1$ times continuously differentiable.)

If $F$ is an $n$-unisolvent set, $A M(f, g)$ denotes the sum of the analytic multiplicities of the zeros of $f-g$. In the case that $F$ is an $n$-phunisolvent set, $A M(f, g)$ is the sum of the analytic multiplicities of the zeros of $f-g$ in the half-open interval $[-\pi, \pi)$.

Proposition 4.6. Let $F$ be an unrestricted n-unisolvent set on the open interval $I$ or an unrestricted $n$-phunisolvent set. Let $f, g, h \in F, f \neq g \neq h \neq f$, and let $x_{0} \in I$ (or $x_{0} \in[-\pi, \pi)$ ) be a zero of $f-g$ of analytic multiplicity $m$ and a zero of $f-h$ of analytic multiplicity $m^{\prime}$. Then

1) $A M(f, g) \leq n-1$. In particular, $m, m^{\prime} \leq n-1$;

2) the function $g-h$ has a zero of multiplicity at least $\min \left\{m, m^{\prime}\right\}$ at $x_{0}$;

3) $x_{0}$ is a nodal zero of $(f-g)^{(m-2 k-1)}$ if $1 \leq 2 k+1 \leq m$ and a non-nodal zero of $(f-g)^{(m-2 k)}$ if $1<2 k \leq m$. In particular, if $m$ is an odd (even) integer, then $x_{0}$ is a (non-)nodal zero of $f-g$.

Proof. We only prove this in the case that $F$ is an unrestricted $n$-unisolvent set on the open interval $I$. That these statements are also true in the case of unrestricted $n$-phunisolvent $F$ can then be deduced as in Corollary 2.3 .

1) If $A M(f, g) \geq n$, there exists an SIC of order $n$ that is satisfied by both $f$ and $g$. This contradicts the fact that $F$ is unrestricted.

2 ) is an immediate consequence of the definition of analytic multiplicity of a zero. 


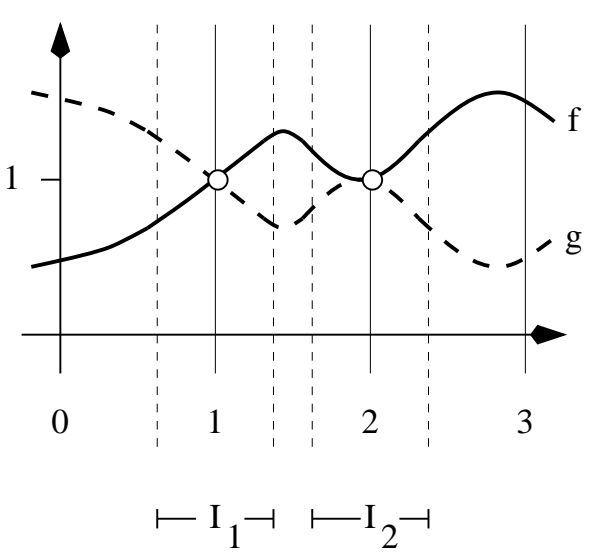

FIGURE 5

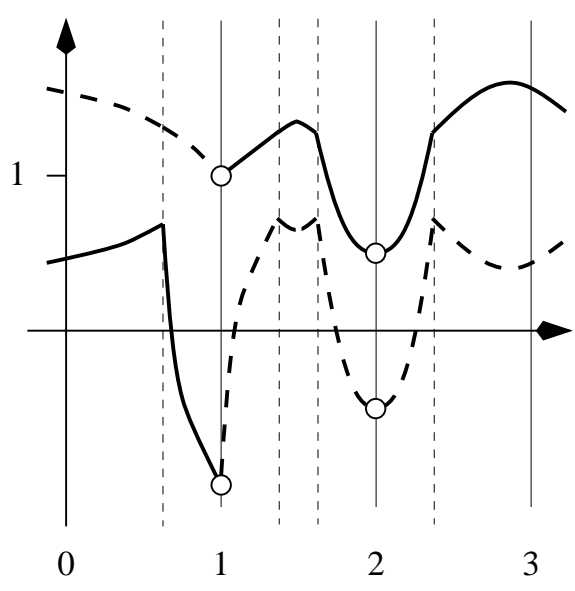

FiguRE 6

3) From the definition of analytic multiplicity it is clear that $(f-g)^{(m)}$ has constant sign in some neighborhood of $x_{0}$. Furthermore,

$$
(f-g)^{(m-1)}(x)=\int_{x_{0}}^{x}(f-g)^{(m)}(t) d t, \quad x \in I .
$$

So, $x_{0}$ is a nodal zero of $(f-g)^{(m-1)}$. If $m>1$, then

$$
(f-g)^{(m-2)}(x)=\int_{x_{0}}^{x}(f-g)^{(m-1)}(t) d t, \quad x \in I .
$$

We conclude that $x_{0}$ is a non-nodal of $(f-g)^{(m-2)}$. Continuing to argue in this manner, we find that $x_{0}$ is a nodal zero of $(f-g)^{(m-2 k-1)}$ if $1 \leq 2 k+1 \leq m$, and a non-nodal zero of $(f-g)^{(m-2 k)}$ if $1<2 k \leq m$.

Let $F$ be an unrestricted $n$-unisolvent set on the open interval $I=(a, b)$ and let $O$ be the flat $n$-OA associated with it. With every SIC $s=\left\{x_{1}, \ldots, x_{k}\left|\lambda_{x_{1}}, \ldots, \lambda_{x_{k}}\right|\right.$ $\left.y_{x_{1}}^{(0)}, \ldots, y_{x_{k}}^{\left(\lambda_{x_{k}}-1\right)}\right\}$ on $I$ of order $m \leq n-1$ we are going to associate a flat $(n-m)$ OA $O_{s}$ that has the same point set as $O$. Let us assume that $x_{1}<x_{2}<\ldots<x_{k}$.

[During our construction it helps to keep one specific example in mind. Remarks about this specific example will always be inserted in square brackets. Let $n=5$, $I=(0,3), k=2, s=\left\{x_{1}=1, x_{2}=2\left|\lambda_{x_{1}}=1, \lambda_{x_{2}}=2\right| y_{x_{1}}^{(0)}=1, y_{x_{2}}^{(0)}=1, y_{x_{2}}^{(1)}=0\right\}$. So, in this case $m=\lambda_{x_{1}}+\lambda_{x_{2}}=3$ and $O_{s}$ will be a 2-OA.]

Let $F_{s}=\left\{f \in F \mid f^{(j)}\left(x_{i}\right)=y_{x_{i}}^{(j)}, i=1,2, \ldots, k, j=0,1, \ldots, \lambda_{x_{i}}-1\right\}$, i.e., $F_{s}$ is the set of all $f \in F$ that satisfy $s$. Let $I^{\prime}=I \backslash\left\{x_{1}, x_{2}, \ldots, x_{k}\right\}$ and let $F_{s}^{\prime}$ be the restriction of $F_{s}$ to $I^{\prime}$. All graphs of functions in $F_{s}$ pass through the points $\left(x_{1}, y_{x_{1}}^{(0)}\right),\left(x_{2}, y_{x_{2}}^{(0)}\right), \ldots,\left(x_{k}, y_{x_{k}}^{(0)}\right)$. We choose $\epsilon_{i}>0, i=1,2, \ldots, k$, such that the $k$ intervals $I_{i}=\left[x_{i}-\epsilon_{i}, x_{i}+\epsilon_{i}\right]$ are disjoint and all contained in $I$.

[Let $f, g \in F_{s}, f \neq g, f^{(1)}(1)=1 \neq-1=g^{(1)}(1), f^{(2)}(2)=1 \neq-1=g^{(2)}(2)$. Then, by Proposition 4.6, $f-g$ has a nodal zero at 1 and a non-nodal zero at 2, see Figure 5 . Notice that in the general case $f-g$ has a (non-)nodal zero at $x_{i}$ if and only if $\lambda_{x_{i}}$ is odd (even).]

It is possible to construct a homeomorphism $\gamma$ of $I^{\prime} \times \mathbb{R}$ that leaves all verticals in this set globally fixed, and if a vertical is not contained in one of the strips $I_{i} \times \mathbb{R}, \gamma$ 


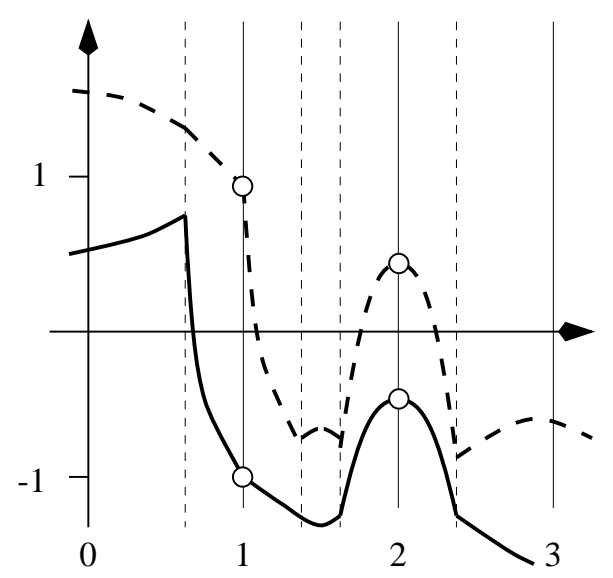

FiguRE 7

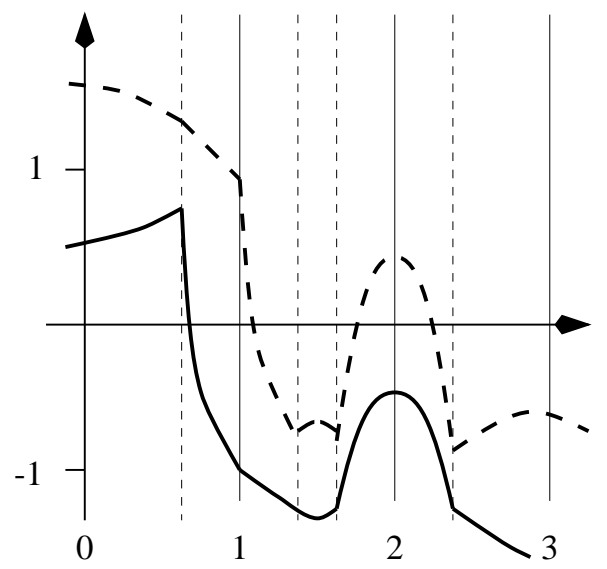

Figure 8

leaves this vertical pointwise fixed. Note that in between two adjacent strips there always exists such a vertical. This means that $\gamma$ is orientation-preserving when restricted to the connected components of $I^{\prime} \times \mathbb{R}$. For arbitrary $\delta_{i}$ with $0<\delta_{i}<\epsilon_{i}$, $i=1,2, \ldots, k$, the homeomorphism $\gamma$ can be constructed in such a way that in $\left(\left(x_{i}-\delta_{i}, x_{i}\right) \cup\left(x_{i}, x_{i}+\delta_{i}\right)\right) \times \mathbb{R}$ it looks as follows:

$$
\gamma(x, y)=\left(x,\left(\operatorname{sgn}\left(x-x_{i}\right)\right)^{\lambda_{x_{i}}} \frac{y-\sum_{j=0}^{\lambda_{x_{i}}-1} y_{x_{i}}^{(j)} \frac{\left(x-x_{i}\right)^{j}}{j !}}{\frac{\left(x-x_{i}\right)^{\lambda_{x_{i}}}}{\lambda_{x_{i}} !}}\right)
$$

Note that the factor $\left(\operatorname{sgn}\left(x-x_{i}\right)\right)^{\lambda_{x_{i}}}$ makes sure that this map is really orientationpreserving. Here $\operatorname{sgn}$ is the function $\mathbb{R} \rightarrow \mathbb{R}$ that maps $x \in \mathbb{R}$ to $-1,0,1$ if $x<0$, $x=0, x>0$, respectively. Furthermore, for $f \in F_{s}^{\prime}$ we find that

$$
\begin{aligned}
\lim _{x \rightarrow x_{i} \pm} \gamma(x, f(x)) & =\left(x_{i},( \pm 1)^{\lambda_{x_{i}}} \lim _{x \rightarrow x_{i} \pm} \frac{f(x)-\sum_{j=0}^{\lambda_{x_{i}}-1} y_{x_{i}}^{(j)} \frac{\left(x-x_{i}\right)^{j}}{j !}}{\frac{\left(x-x_{i}\right)^{\lambda_{x_{i}}}}{\lambda_{x_{i}} !}}\right) \\
& =\left(x_{i},( \pm 1)^{\lambda_{x_{i}}} f^{\left(\lambda_{x_{i}}\right)}\left(x_{i}\right)\right) .
\end{aligned}
$$

[Let us have a look at what is happening to our example; see Figure 6. We note that if we flip both the strips $(1,2) \times \mathbb{R}$ and $(2,3) \times \mathbb{R}$ around the $x$-axis, both the dotted line and the solid line can be completed to continuous curves by adding two points; see Figures 7 and 8 ]

As in our example, the images of all graphs of functions in $F_{s}$ under $\gamma$ are still discontinuous. But by starting from the left and working towards the right we can match up the ends on both sides of the verticals $\left\{x_{i}\right\} \times \mathbb{R}$ by flipping connected components of $I^{\prime} \times \mathbb{R}$ around the $x$-axis whenever necessary. This flipping operation amounts to an involutory homeomorphism $\gamma^{\prime}$ of $I^{\prime} \times \mathbb{R}$. Let $s i g_{i}=\prod_{j=1}^{i}(-1)^{\lambda_{x_{j}}}$ for $i=1,2, \ldots, k$, and let $x_{k+1}=b$. Then $\gamma^{\prime}$ looks as follows:

$$
\gamma^{\prime}: I^{\prime} \times \mathbb{R} \rightarrow I^{\prime} \times \mathbb{R}:(x, y) \mapsto \begin{cases}(x, y) & \text { for } a<x<x_{1} \\ \left(x, \text { sig }_{i} y\right) & \text { for } x_{i}<x<x_{i+1}, i=1,2, \ldots, k\end{cases}
$$


The image of a graph of a function in $F_{s}^{\prime}$ under $\gamma^{\prime} \gamma$ can be completed to a continuous curve by adding to it all its boundary points in $I \times \mathbb{R}$ (see Figure 8). Let $L_{s}$ be the set of all curves that have been completed in this manner. Let $O_{s}=\left(I \times \mathbb{R}, L_{s}, \|\right)$.

Proposition 4.7. $O_{s}$ is a flat $(n-m)-O A$.

Proof. Let $E$ be the set of continuous functions that corresponds to $O_{s}$. We have to show that $E$ is an $(n-m)$-unisolvent set on $I$, i.e., we have to show that for $n-m$ pairwise non-parallel points $\left(\bar{x}_{1}, y_{\bar{x}_{1}}\right),\left(\bar{x}_{2}, y_{\bar{x}_{2}}\right), \ldots,\left(\bar{x}_{n-m}, y_{\bar{x}_{n-m}}\right)$ there exists a uniquely determined function in $E$ interpolating these points. We translate this problem to an equivalent problem which consists in showing that for a certain SIC $\bar{s}$ of order $n$ containing $s$ there exists exactly one element in the original $n$ unisolvent set $F$ that satisfies it. Such a unique solution always exists by definition. This then proves the proposition.

From our construction of $O_{s}$ above it is clear how to construct $\bar{s}$. W.l.o.g. we may assume that precisely the first $l, 1 \leq l \leq m-n$, of the $\bar{x}_{i}$ s are contained among the $x_{j} \mathrm{~S}$ in $s$. Remember that $s=\left\{x_{1}, \ldots, x_{k}\left|\lambda_{x_{1}}, \ldots, \lambda_{x_{k}}\right| y_{x_{1}}^{(0)}, \ldots, y_{x_{k}}^{\left(\lambda_{x_{k}}-1\right)}\right\}$ was an abbreviation for $s=(X, \Lambda, Y)$, where $X=\left\{x_{1}, x_{2}, \ldots, x_{k}\right\}, \Lambda=\left\{\lambda_{x_{1}}, \lambda_{x_{2}}, \ldots, \lambda_{x_{k}}\right\}$ and $Y=\left\{Y_{x_{1}}, Y_{x_{2}}, \ldots, Y_{x_{k}}\right\}$ such that $Y_{x_{i}}=\left\{y_{x_{i}}^{(0)}, y_{x_{i}}^{(1)}, \ldots, y_{x_{i}}^{\left(\lambda_{x_{i}}-1\right)}\right\}, i=1,2, \ldots$, $k$. Now let $\bar{s}=(\bar{X}, \bar{\Lambda}, \bar{Y})$, where $\bar{X}=X \cup\left\{\bar{x}_{l+1}, \bar{x}_{l+2}, \ldots, \bar{x}_{n-m}\right\}, \Lambda=\left\{\bar{\lambda}_{x_{1}}, \bar{\lambda}_{x_{2}}\right.$, $\left.\ldots, \bar{\lambda}_{x_{k}}, \bar{\lambda}_{\bar{x}_{l+1}}, \bar{\lambda}_{\bar{x}_{l+2}}, \ldots, \bar{\lambda}_{\bar{x}_{n-m}}\right\}$, where $\bar{\lambda}_{\bar{x}_{l+1}}=\bar{\lambda}_{\bar{x}_{l+2}}=\ldots=\bar{\lambda}_{\bar{x}_{n-m}}=1$ and

$$
\bar{\lambda}_{x_{i}}= \begin{cases}\lambda_{x_{i}}+1 & \text { if } x_{i} \text { is one of the } \bar{x}_{j} \mathrm{~s}, \\ \lambda_{x_{i}} & \text { otherwise. }\end{cases}
$$

Finally, $\bar{Y}=\left\{\bar{Y}_{x_{1}}, \bar{Y}_{x_{2}}, \ldots, \bar{Y}_{x_{k}}, \bar{Y}_{\bar{x}_{l+1}}, \bar{Y}_{\bar{x}_{l+2}}, \ldots, \bar{Y}_{\bar{x}_{n-m}}\right\}$ such that $\bar{Y}_{x_{i}}=\left\{y_{x_{i}}^{(0)}, y_{x_{i}}^{(1)}\right.$, $\left.\ldots, y_{x_{i}}^{\left(\bar{\lambda}_{x_{i}}-1\right)}\right\}, i=1,2, \ldots, k$, where

$$
y_{x_{i}}^{\left(\bar{\lambda}_{x_{i}}-1\right)}= \begin{cases}\operatorname{sig}_{i} y_{x_{i}} & \text { if } x_{i} \text { is one of the } \bar{x}_{j} \mathrm{~s} \\ y_{x_{i}}^{\left(\lambda_{x_{i}}-1\right)} & \text { otherwise }\end{cases}
$$

and

$$
\bar{Y}_{\bar{x}_{l+j}}= \begin{cases}\left\{y_{\bar{x}_{l+j}}\right\} & \text { if } a<\bar{x}_{l+j}<x_{1}, \\ \left\{\operatorname{sig}_{i} y_{\bar{x}_{l+j}}\right\} & \text { if } x_{i}<\bar{x}_{l+j}<x_{i+1},\end{cases}
$$

for $j=1,2, \ldots, n-m-l$.

[We demonstrate how this translation works in our specific example.

[In our example $n=5$ and $m=3$. We have to show that, given two non-parallel points $\left(\bar{x}_{3}, y_{\bar{x}_{3}}\right),\left(\bar{x}_{4}, y_{\bar{x}_{4}}\right)$, there exists exactly one function in $E$ that interpolates both points. If neither $\bar{x}_{3}$ nor $\bar{x}_{4}$ coincides with $x_{1}$ or $x_{2}$, let $\left(\bar{x}_{3}, \bar{y}_{\bar{x}_{3}}\right),\left(\bar{x}_{4}, \bar{y}_{\bar{x}_{4}}\right)$ be the images of $\left(\bar{x}_{3}, y_{\bar{x}_{3}}\right)$ and $\left(\bar{x}_{4}, y_{\bar{x}_{4}}\right)$, respectively, under the homeomorphism $\left(\gamma^{\prime} \gamma\right)^{-1}$. Then our problem consists in showing that the SIC $\left\{x_{1}, x_{2}, \bar{x}_{3}, \bar{x}_{4} \mid \bar{\lambda}_{x_{1}}=\right.$ $\left.1, \bar{\lambda}_{x_{2}}=2, \bar{\lambda}_{\bar{x}_{3}}=1, \bar{\lambda}_{\bar{x}_{4}}=1 \mid y_{x_{1}}^{(0)}, y_{x_{2}}^{(0)}, y_{x_{2}}^{(1)}, y_{\bar{x}_{3}}^{(0)}=\bar{y}_{\bar{x}_{3}}, y_{\bar{x}_{4}}^{(0)}=\bar{y}_{\bar{x}_{4}}\right\}$ is satisfied by exactly one $f \in F$. This is certainly true, since this SIC is of order 5 and $F$ is an unrestricted 5-unisolvent set.

[If $\bar{x}_{4}$ coincides with $x_{1}$ and $\bar{x}_{3}$ does not coincide with $x_{2}$, let again $\left(\bar{x}_{3}, \bar{y}_{\bar{x}_{3}}\right)$ be the image of $\left(\bar{x}_{3}, y_{\bar{x}_{3}}\right)$ under the homeomorphism $\left(\gamma^{\prime} \gamma\right)^{-1}$. Then our problem consists in showing that the SIC $\left\{x_{1}, x_{2}, \bar{x}_{3}\left|\bar{\lambda}_{x_{1}}=2, \bar{\lambda}_{x_{2}}=2, \bar{\lambda}_{x_{3}}=1\right| y_{x_{1}}^{(0)}, y_{x_{1}}^{(1)}=\right.$ $\left.-y_{\bar{x}_{4}}, y_{x_{2}}^{(0)}, y_{x_{2}}^{(1)}, y_{\bar{x}_{3}}^{(0)}=\bar{y}_{\bar{x}_{3}}\right\}$ of order 5 is satisfied by exactly one $f \in F$. This is certainly true. 
[The remaining two cases are dealt with in a similar fashion.]

Let us go back, for the moment, to general nested $n$-phOAs. Deriving at a point $p$ means concentrating on the circles through $p$, removing the parallel class $p$ is contained in, "twisting" the strip $(-\pi, \pi) \times \mathbb{R}$, i.e., flipping the strip on just one side, in order to move from cylinder to Möbius strip or the other way around, and finally gluing in another parallel class to fit the left and right sides of the strip together. It is convenient to "identify" the parallel class that gets removed with the parallel class that gets added. In this way it makes sense to say: "We derive at $m$ points of the same parallel class", i.e., we choose a point and derive, choose another point and derive again, and so on, $m$ times. All this sounds very much like what we just did.

So, let $F$ be an unrestricted $n$-phunisolvent set and let $O$ be the $n$-phOA associated with it. Let $s=\left\{x_{1}, \ldots, x_{k}\left|\lambda_{x_{1}}, \ldots, \lambda_{x_{k}}\right| y_{x_{1}}^{(0)}, \ldots, y_{x_{k}}^{\left(\lambda_{x_{k}}-1\right)}\right\}$ be an SIC of order $m \leq n-1$ on $[-\pi, \pi)$. Then we can construct an $(n-m)$-phOA in exactly the same manner as before when $F$ was an unrestricted $n$-unisolvent set. The only difference in our picture is that the left and right sides of the interval $I=[-\pi, \pi]$ get identified and therefore, when we are busy flipping the strips, we might actually have to introduce a twist at the last flip in order to be able to glue respective sides together (thereby forming a Möbius strip). Notice that odd $\lambda_{x_{i}}$ s introduce a flip (or un-flip) and every even $\lambda_{x_{i}}$ s introduces no flip at all. Furthermore, if $n-m=n-\left(\lambda_{x_{1}}+\lambda_{x_{2}}+\ldots+\lambda_{x_{k}}\right)$ is even, we end up with a Möbius strip, and if $n-m$ is odd, with a cylinder.

Now, clearly, if $O$ is nested, then for all $p=\left(x_{1}, y_{1}\right) \in[-\pi, \pi) \times \mathbb{R}$ and $s=$ $\left\{x_{1}\left|\lambda_{x_{1}}=1\right| y_{x_{1}}^{(0)}=y_{1}\right\}, O_{s}$ is the derived $(n-1)$-phOA at the point $p$. We already know that $O_{s}$ is an $(n-1)$-phOA. We just have to check that it is nested. The possible candidates for all the derived $(n-2)$-phOAs of this $(n-1)$-phOA are the $(n-2)$-phOAs that correspond to SICs of order 2 on $[-\pi, \pi)$ that contain $s$. Again it remains to check that all these $(n-2)$-phOAs are nested. Let $s^{\prime}$ be an SIC of order 2 that contains $s$. Then the possible candidates for all the derived (nested) $(n-3)$-phOAs of the $(n-2)$-phOA associated with $s^{\prime}$ are the $(n-3)$-phOAs that correspond to SICs of order 3 on $[-\pi, \pi)$ that contain $s^{\prime}$. Again it remains to check that all these $(n-3)$-phOAs are nested. We can continue to argue in this manner until we are left with 1-phOAs, which are automatically nested. This, of course, shows that all the $(n-m)$-phOAs we came across on the way are nested themselves. In particular we have proved

Proposition 4.8. The n-phOA that corresponds to an unrestricted $n$-phunisolvent set, $n \geq 1$, is nested.

Similarly,

Proposition 4.9. The flat $n$-OA that corresponds to an unrestricted $n$-unisolvent set, $n \geq 1$, is nested.

Corollary 4.10. a) The 3-phOA that corresponds to an unrestricted 3-phunisolvent set is a flat Laguerre plane.

b) The n-phOA that corresponds to an n-phunisolvent set, all of whose elements are $n-1$ times continuously differentiable, and which has the property of unique $n$ initial values, is nested. In particular, the 3-phOA that corresponds to a periodic 
3-unisolvent set, all of whose elements are twice continuously differentiable, and which has the property of unique 3 initial values, is a flat Laguerre plane.

c) The flat n-OA that corresponds to an n-unisolvent set on an open interval, all of whose elements are $n-1$ times continuously differentiable, and which has the property of unique $n$ initial values, is nested.

Proof. a) is an immediate consequence of Propositions 3.5 and 4.8. b) follows from a) and Corollary 4.4. c) follows from Propositions 4.3 and 4.9.

Corollary 4.11. For all $n \in \mathbb{N}$ there exist nested $n$-(ph)OAs.

Proof. This is a consequence of Propositions 4.8 and 4.9 and Examples 4.1.1 and 4.5.1. Remember also that $\operatorname{span}\left\{1, x, x^{2}, \ldots, x^{n-1}\right\}$ is an unrestricted $n$-unisolvent set on any interval.

4.12. Remarks. a) Just because all elements of an $n$-(ph)unisolvent set $F$ are $n-1$ times continuously differentiable, it does not necessarily follow that $F$ is unrestricted. Take, for example, the set of functions $\mathbb{R} \rightarrow \mathbb{R}: x \mapsto a x^{3}+b, a, b \in \mathbb{R}$. This set is 2-unisolvent on $\mathbb{R}$ and all its elements are continuously differentiable. Nevertheless the SIC $\left\{x_{1}=0\left|\lambda_{x_{1}}=2\right| y_{x_{1}}^{(0)}=0, y_{x_{1}}^{(1)}=1\right\}$ is not satisfied by any element of the set.

The flat 2-OA that corresponds to this 2-unisolvent set on $\mathbb{R}$ is a flat (dually) affine plane. As we have seen in Section 1, any flat (dually) affine plane can be extended to a Möbius strip plane. Therefore, as a corollary of Proposition 3.1, we have that every flat dually affine plane is in fact a nested flat 2-OA.

b) Unrestricted $n$-(ph)unisolvent sets can be interpreted as sets that solve the Hermite interpolation problems. The more general type of Hermite-Birkhoff problems has not been investigated for general $n$-unisolvent sets (see [11] for general information about Hermite-Birkhoff interpolation).

c) We note that in our examples of nested $n$-(ph)OAs, $n \geq 3$, the order in which we derive at certain points does not change the result. For example, given two points $p_{1}$ and $p_{2}$ in the point set of one of our examples, we can first derive at $p_{1}$ and then at $p_{2}$, or first at $p_{2}$ and then at $p_{1}$. The resulting $(n-2)$-OAs will always be topologically equivalent. It is possible to establish a similar result for general nested $n$-(ph)OAs. It is then possible to define the multiplicity of a zero of $f-g$, $f, g \in F$, without having to resort to derivatives.

d) Special kinds of finite $n$-OAs whose definitions come close to that of nested $n$-OAs have been investigated in, e.g., [8], [28], [29].

e) In [17] (see also [18]) we proved, essentially, that nested 3-(ph)OAs can be constructed by "integrating" nested 2-(ph)OAs. A generalization of this construction principle to nested $n$-(ph)OAs is possible.

f) Topological incidence geometers have proved that the groups of automorphisms of flat affine planes, Möbius strip planes and flat Laguerre planes are finitedimensional Lie groups all of whose elements are homeomorphisms of the respective point spaces. Topological incidence geometers have been very successful in classifying the examples of these three different structures according to the dimension of their automorphism groups. Generalizations of these results are possible for nested $n$-(ph)OAs.

Topological incidence geometers also investigate 2-unisolvent sets of continuous functions $\mathbb{R}^{m} \rightarrow \mathbb{R}^{m}, m=2,4,8$, that correspond to 4-, 8- and 16-dimensional projective planes, and 3-unisolvent sets of continuous functions $\mathbb{R}^{2} \rightarrow \mathbb{R}^{2}$ that 
correspond to 4-dimensional Laguerre planes. Again it can be proved that the groups of continuous automorphisms of all these structures are finite-dimensional Lie groups. One can then proceed, as in the case $m=1$, to classify these incidence structures according to the dimension of their automorphism groups. A generalization of all these results and our results for $n$-unisolvent sets of continuous functions $\mathbb{R}^{2} \rightarrow \mathbb{R}^{2}$ seems to be possible to some extent. See [21], [22], [1], [26] for details about higher-dimensional affine planes and Laguerre planes.

We will elaborate on these remarks in a follow-up to this paper.

\section{REFERENCES}

1. D. Betten, Topologische Geometrien auf dem Möbiusband, Math. Z. 107 (1968), 363-379. MR 38:6607

2. Ph. C. Curtis, Jr., n-parameter families and best approximation, Pacific J. Math. 9 (1959), 1013-1027. MR 21:7385

3. W. Forst, Variationsmindernde Eigenschaften eines speziellen Kreinschen Kernes, Math. Z. 148 (1976), 67-70. MR 53:5886

4. D. G. Glynn, A geometrical representation theory for orthogonal arrays, Bull. Austr. Math. Soc. 49 (2) (1994), 311-324. MR 94m:05039

5. H. Groh, Topologische Laguerreebenen I, Abh. Math. Sem. Univ. Hamburg 32 (1968), 216231. MR 38:2660

6. H. Groh, Topologische Laguerreebenen II, Abh. Math. Sem. Univ. Hamburg 34 (1970), 11-21. MR 41:2506

7. P. Hartman, Unrestricted n-parameter families, Rend. Circ. Mat. Palermo (2) 7 (1958), 123142. MR 21:4211

8. W. Heise, Optimal codes, n-arcs and Laguerre geometry, Acta Informatica 6 (1976), 403-406. MR 54:12356

9. D. J. Johnson, The trigonometric Hermite-Birkhoff interpolation problem, Trans. Amer. Math. Soc. 212 (1976), 365-374. MR 54:5712

10. S. Karlin - W. J. Studden, Tchebycheff Systems with Applications in Analysis and Statistics, Interscience, New York, 1966. MR 34:4757

11. G. G. Lorentz - K. Jetter - S. D. Riemenschneider, Birkhoff interpolation, Addison-Wesley, Reading, MA, 1983, and Cambridge University Press, New York, 1984. MR 84g:41002

12. R. A. Lorentz, Simultaneous Approximation and Birkhoff Interpolation II: The periodic case, J. Approx. Theory 44 (1985), 21-29. MR 86j:41018

13. R. A. Lorentz, Some new periodic Chebysheff Subspaces, J. Approx. Theory 61 (1990), 13-22. MR 81a:41006

14. R. M. Mathsen, $\lambda(n)$-parameter families, Canad. Math. Bull. 12 (1969), 185-191. MR 39:7191

15. T. S. Motzkin, Approximation by curves of a unisolvent family, Bull. Amer. Math. Soc. 55 (1949), 789-793. MR 11:101f

16. F. R. Moulton, A simple non-desarguesian plane geometry, Trans. Amer. Math. Soc. 3 (1902), 192-195.

17. B. Polster, Integrating and differentiating two-dimensional incidence structures, Arch. Math 64 (1995), 75-85. MR 95i:51015

18. B. Polster, Integrating completely unisolvent functions, J. Approx. Theory 82 (1995), 434-439. MR 96g: 26016

19. J. R. Rice, The Approximation of Functions I, Addison-Wesley, Reading, MA, 1964. MR 29:3795

20. J. R. Rice, The Approximation of Functions II, Addison-Wesley, Reading, MA, 1969. MR 39:5989

21. H. Salzmann, Topological planes, Adv. Math. 2 (1967), 1-60. MR 36:3201

22. H. Salzmann - D. Betten - T. Grundhöfer - H. Hähl - R. Löwen - M. Stroppel, Compact projective planes, de Gruyter, Berlin, 1995. MR 97b:51009

23. L. L. Schumaker, Spline functions: Basic theory, Wiley, New York, 1981. MR 82j:41001

24. G. F. Steinke, Topological affine planes composed of two Desarguesian halfplanes and projective planes with trivial collineation group, Arch. Math. 44 (1985), 472-480. MR 86j:51023 
25. G. F. Steinke, Semiclassical topological flat Laguerre planes obtained by pasting along two parallel classes, J. Geom. 32 (1988), 133-156. MR 89k:51021

26. G. F. Steinke, Topological circle geometries, in: Handbook of Incidence Geometry (F. Buekenhout, ed.), Elsevier, pp. 1325-1353, 1995. MR 96i:51015

27. L. Tornheim, On n-parameter families of functions and associated convex functions, Trans. Amer. Math. Soc. 69 (1950), 457-467. MR 12:395d

28. M. L. H. Willems, Optimal codes, Laguerre and special Laguerre i-structures, Eur. J. Comb. 4 (1983), 87-92. MR 85a:51007

29. M. L. H. Willems - J. A. Thas, A note on the existence of special Laguerre i-structures and optimal codes, Eur. J. Comb. 4 (1983), 93-96. MR 85a:51008

Department of Pure Mathematics, The University of Adelaide, Adelaide, SA 5005, Australia

E-mail address: bpolster@maths.adelaide.edu.au 